\title{
Light-Emitting Diodes with Manganese Halide Tetrahedron Embedded in Anti-Perovskites
}

Siyu Yan, ${ }^{1}$ Kaixin Tang, ${ }^{1}$ Yuejian Lin, ${ }^{2}$ Yuanhang Ren, ${ }^{2}$ Wanli Tian, ${ }^{1}$ Hua Chen, ${ }^{1}$ Tingting Lin, ${ }^{3}$ Longzhen Qiu, ${ }^{4}$ Xiaoyong Pan $^{5}$ and Weizhi Wang*I

${ }^{1}$ State Key Laboratory of Molecular Engineering of Polymers, Collaborative Innovation Center of Polymers and Polymer Composite Materials, Department of Macromolecular Science, Fudan University, Shanghai 200433, P. R. China

${ }^{2}$ Advanced Materials Laboratory, Department of Chemistry, Fudan University, Shanghai 200433, P. R. China

3Institute of Materials Research and Engineering, A*STAR (Agency for Science, Technology and Research), 2 Fusionopolis Way, Innovis, Singapore 138634, Singapore

${ }^{4}$ National Engineering Lab of Special Display Technology, State Key Lab of Advanced Display Technology, Academy of Opto-Electronic Technology, Hefei University of Technology, Hefei 230009, China

${ }^{5}$ School of Material \& Science Engineering, Nanyang Technological University, 62 Nanyang Drive, Singapore 637459, Singapore 


\section{Supporting Information}

\section{Experimental Section}

\section{Materials and chemicals}

Cesium chloride ( $\mathrm{CsCl}, 99.9 \%$, metal basis), Cesium bromide ( $\mathrm{CsBr}, 99.5 \%$, metals basis), Cesium iodide (CsI, 99.9\%, metal basis), Lithium fluoride (LiF, $99.99 \%$, metals basis) and 2,2',2”(1,3,5-benzinetriyl)-tris(1-phenyl-1-H-benzimidazole) $(\mathrm{TPBi},>99.5 \%)$ were purchased from Aladdin Reagents. Anhydrous manganese chloride $\left(\mathrm{MnCl}_{2}, \mathrm{AR}\right)$ and anhydrous manganese bromide $\left(\mathrm{MnBr}_{2}, \mathrm{AR}\right)$ were purchased from Changsha Jingkang New Material Technology. Patterned ITO-glass substrates (sheet resistance, $15 \mathrm{~V} \mathrm{sq}^{-1}$; ITO thickness, $100 \mathrm{~nm}$ ) were purchased from Luoyang Guluo Glass Company. PEDOT: PSS (4083, $1.3 \sim 1.7$ wt \% dispersion in $\left.\mathrm{H}_{2} \mathrm{O}\right)$ was purchased from Xi'an Polymer Light Technology Corp. Isopropyl alcohol (AR) and aluminum wire $(\Phi 1 \mathrm{~mm}, 5 \mathrm{~N})$ were purchased from Sinopharm Chemical Reagent. Other chemicals, without stated, were purchased from Sigma-Aldrich. All materials were used as received without further purification.

\section{Preparation of perovskite single crystal}

All samples 1-6 were synthesized via a traditional crystal growth method, solid-state reaction method in vacuum. For 1-6, $2.46 \mathrm{mmol} \mathrm{CsCl}(0.4142 \mathrm{~g})$ and $0.82 \mathrm{mmol} \mathrm{MnCl}_{2}(0.1032 \mathrm{~g}), 2.46$ $\mathrm{mmol} \mathrm{CsCl}(0.4142 \mathrm{~g})$ and $0.82 \mathrm{mmol} \mathrm{MnBr}_{2}(0.1762 \mathrm{~g}), 2.46 \mathrm{mmol} \mathrm{CsBr}(0.5238 \mathrm{~g})$ and 0.82 $\mathrm{mmol} \mathrm{MnCl}_{2}(0.1032 \mathrm{~g}), 2.46 \mathrm{mmol} \mathrm{CsBr}(0.5238 \mathrm{~g})$ and $0.82 \mathrm{mmol} \mathrm{MnBr}_{2}(0.1762 \mathrm{~g}), 2.46 \mathrm{mmol}$ CsI (0.6391 g) and $0.82 \mathrm{mmol} \mathrm{MnCl} 2(0.1032 \mathrm{~g}), 2.46 \mathrm{mmol} \mathrm{CsI}(0.6391 \mathrm{~g})$ and $0.82 \mathrm{mmol} \mathrm{MnBr} 2$ $(0.1762 \mathrm{~g})$ were weighed, ground and sealed in a quartz tube in vacuum at a stoichiometric molar 
ratio of 3:1, separately. The as-prepared quartz tubes were then heated in a tube furnace with single temperature zone to $720^{\circ} \mathrm{C} \pm 50^{\circ} \mathrm{C}$ with temperature gradient of $5^{\circ} \mathrm{C} / \mathrm{min}$, maintained at $720^{\circ} \mathrm{C} \pm 50$ ${ }^{\circ} \mathrm{C}$ for 120 min (to make sure the raw materials in the molten state) and then cooled down to room temperature at a rate of $-0.2{ }^{\circ} \mathrm{C} / \mathrm{min}$, pure single crystals $\mathbf{1 - 6}$ can be obtained. After synthesis, the ampoule was cut open in an Ar glovebox to yield small crystals. All characterizations were carried out in air-free conditions given that the obtained materials were hygroscopic and sensitive to air. CCDC deposition number, 2010943 (single crystal 4), 2047511-2047515 (single crystals 1-3, 56).

\section{Characterizations of single crystals}

Single crystal X-ray diffraction data of single crystals 1-6 were collected using Bruker D8 Venture single crystal X-ray diffractometer. UV-vis absorption spectrum (UV) was measured by the UVvisible spectrophotometer (Lambda 750, Perkin Elmer) at room temperature. Steady-state photoluminescence spectra and photoluminescence lifetimes were measured by FLS1000 spectrofluorometer (Edinburgh Instruments) from room temperature and $77 \mathrm{~K}$ (liquid nitrogen was used to cool the samples). Temperature-dependent PL spectra were conducted on a GZtek (Shanghai) R\&D Centre Fiber Spectrometer with a homemade temperature control enclosure. Photoluminescence quantum yield was recorded on a Photo Technology International, Inc. QM40 by means of a time-corrected single photon counting system at room temperature with an integrating sphere. Thermogravimetric analysis (TGA) and differential scanning calorimetry (DSC) analysis of the single crystal were evaluated using a synchronous thermal analysis-mass spectrometry system (SDT Q600-GSD $301 \mathrm{~T} 2$, TA-Pfeiffer) at a heating rate of $10{ }^{\circ} \mathrm{C} / \mathrm{min}$ under $\mathrm{N}_{2}$ atmosphere. X-ray photoelectron spectroscopy (XPS) results were collected by a PHI 5000 
equipped with a dual anode X-ray source. Spectra background was fit and subtracted by an integrated Shirley function. XPS curves were deconvoluted using Gaussian peak functions for the halides.

\section{Device fabrication}

Patterned ITO glasses $(20 \mathrm{~mm} \times 20 \mathrm{~mm})$ were cleaned as previous report. ${ }^{6}$ PEDOT: PSS solutions were spin-coated on the ITO-coated glass substrates at 4,000 r.p.m. for $60 \mathrm{~s}$ and baked at $140{ }^{\circ} \mathrm{C}$ for $20 \mathrm{~min}$. After cooling to room temperature, anti-perovskite emission layer was deposited using a double-source thermal evaporation system under a high vacuum of $\sim 6 \times 10^{-5}$ torr. $50 \mu \mathrm{L}$ of PMMA solution (relative molecular mass 60,000-100,000, $2 \mathrm{wt} \%$ in toluene) was spin-coated onto the as-formed emissive layer at 4,000 r.p.m. for $60 \mathrm{~s}$ without annealing process to form a 2-nm thin film. A 40-nm-thick layer of TPBi were deposited using a thermal evaporation system under a high vacuum of $\sim 6 \times 10^{-5}$ torr, separately. LiF/Al electrodes were thermally evaporated through a shadow mask under a high vacuum $\sim 6 \times 10^{-7}$ torr. The devices were encapsulated in the cover glass using ultraviolet-curable resin.

\section{$\underline{\text { Device characterization }}$}

The thickness of films was measured using a KLA-Tencor AlphaStep D-600 stylus profiler. Atomic force microscope (AFM) images were acquired with a Digital Instruments NanoScope IV (Bruker Multimode 8, America) operating in the tapping mode. Cross section device and energy dispersive spectrometer (EDS) mages were collected by a Zeiss Gemini SEM500 field-emission scanning electron microscope with Aztec X-Max Extreme energy-dispersive X-ray spectroscopy (EDS) system. The powder X-ray diffraction analysis was measured using Bruker D2 X-ray 
Diffractometer with $\mathrm{Cu} \mathrm{K} \alpha$ radiation. The diffraction pattern angular range was 5-50 degrees (20) with a step size of $0.02^{\circ}$ in the room temperature. Calculated powder patterns were simulated by Mercury software using the crystallographic information files (CIFs) from single-crystal X-ray experiments.

\section{Device measurement}

The fabricated devices were tested in the glovebox. Effective light emitting area of LED is 3-4 $\mathrm{mm}^{2}$. Current-voltage -brightness curves were recorded on a Keithley 2400 unit as well as a Luminance meter, with ITO anode positive bias and aluminum cathode negative bias. The capacitance-voltage measurements were performed using a Keithley 4200 Semiconductor Characterization System and a shielded probe station at a constant frequency of $1000 \mathrm{~Hz}$. The absolute energy spectrum of electroluminescence is achieved by installing an Ocean Optics USB4000 spectrometer on the integrating sphere wall. The EQEs are then calculated from the measured absolute power spectra (mW; Integral time: $1 \mathrm{~s}$; Integral wavelength: 400-700 nm) and the current density. 
Table S1 Parameters of existing compounds on a par with anti-perovskite structure with $\left[\mathrm{MX}_{4}\right] \mathrm{XA}_{3}$ formula.

\begin{tabular}{|c|c|c|c|c|c|c|c|c|}
\hline Compound & $\begin{array}{l}\text { Space } \\
\text { group }\end{array}$ & $r_{M}$ & $r$ & $r_{B}$ & $r_{A}$ & $\mu$ & $g$ & Refs. \\
\hline$\left[\mathrm{HgBr}_{4}\right] \mathrm{BrCs}_{3}{ }^{a}$ & Pnma & 1.02 & 2.43316 & 1.96 & 1.88 & 1.042553 & 0.633635 & 1 \\
\hline$\left[\mathrm{HgCl}_{4}\right] \mathrm{ClCs}_{3}{ }^{a}$ & Pnma & 1.02 & 2.310685 & 1.81 & 1.88 & 0.962766 & 0.626202 & 2 \\
\hline$\left[\mathrm{HgI}_{4}\right] \mathrm{ICs}_{3}{ }^{a}$ & Pbca & 1.02 & 2.629119 & 2.2 & 1.88 & 1.170213 & 0.644392 & 3 \\
\hline$\left[\mathrm{CdBr}_{4}\right] \mathrm{BrCs}_{3}{ }^{a}$ & $\mathrm{I} 4 / \mathrm{mcm}$ & 0.95 & 2.376005 & 1.96 & 1.88 & 1.042553 & 0.618751 & 4 \\
\hline$\left[\mathrm{CdI}_{4}\right] \mathrm{ICs}_{3}{ }^{a}$ & Pnma & 0.95 & 2.571964 & 2.2 & 1.88 & 1.170213 & 0.630383 & 3 \\
\hline$\left[\mathrm{PdF}_{4}\right] \mathrm{FRb}_{3}{ }^{a}$ & $\mathrm{P} 4 / \mathrm{mbm}$ & 0.86 & 1.788128 & 1.33 & 1.72 & 0.773256 & 0.586271 & 5 \\
\hline$\left[\mathrm{PdF}_{4}\right] \mathrm{FCs}_{3}{ }^{a}$ & $\mathrm{P} 4 / \mathrm{mbm}$ & 0.86 & 1.788128 & 1.33 & 1.88 & 0.707447 & 0.557049 & 6 \\
\hline$\left[\mathrm{MnCl}_{4}\right] \mathrm{ClCs}_{3}{ }^{a}$ & $\mathrm{I} 4 / \mathrm{mcm}$ & 0.83 & 2.155551 & 1.81 & 1.88 & 0.962766 & 0.58416 & 7 \\
\hline$\left[\mathrm{MnBr}_{4}\right] \mathrm{BrRb}_{3}{ }^{a}$ & $\mathrm{I} 4 / \mathrm{mcm}$ & 0.83 & 2.278025 & 1.96 & 1.72 & 1.139535 & 0.619029 & 8 \\
\hline$\left[\mathrm{MnBr}_{4}\right] \mathrm{BrCs}_{3}{ }^{a}$ & $\mathrm{I} 4 / \mathrm{mcm}$ & 0.83 & 2.278025 & 1.96 & 1.88 & 1.042553 & 0.593236 & 9 \\
\hline$\left[\mathrm{MnI}_{4}\right] \mathrm{ICs}_{3}{ }^{a}$ & $\mathrm{I} 4 / \mathrm{mcm}$ & 0.83 & 2.473985 & 2.2 & 1.88 & 1.170213 & 0.606369 & 3 \\
\hline$\left[\mathrm{FeCl}_{4}\right] \mathrm{ClTl}_{3}{ }^{a}$ & $\mathrm{I} 4 / \mathrm{mcm}$ & 0.78 & 2.114726 & 1.81 & 1.7 & 1.064706 & 0.602486 & 9 \\
\hline$\left[\mathrm{FeBr}_{4}\right] \mathrm{BrCs}_{3}{ }^{a}$ & $\mathrm{I} 4 / \mathrm{mcm}$ & 0.78 & 2.237201 & 1.96 & 1.88 & 1.042553 & 0.582604 & 9 \\
\hline$\left[\mathrm{ZnCl}_{4}\right] \mathrm{ClCs}_{3}{ }^{a}$ & $\mathrm{I} 4 / \mathrm{mcm}$ & 0.74 & 2.082066 & 1.81 & 1.88 & 0.962766 & 0.564246 & 10 \\
\hline$\left[\mathrm{ZnBr}_{4}\right] \mathrm{BrRb}_{3}{ }^{a}$ & Pnma & 0.74 & 2.204541 & 1.96 & 1.72 & 1.139535 & 0.59906 & 11 \\
\hline$\left[\mathrm{ZnBr}_{4}\right] \mathrm{BrCs}_{3}{ }^{a}$ & $\mathrm{I} 4 / \mathrm{mcm}$ & 0.74 & 2.204541 & 1.96 & 1.88 & 1.042553 & 0.574099 & 12 \\
\hline$\left[\mathrm{ZnI}_{4}\right] \mathrm{ICs}_{3}{ }^{a}$ & Pnma & 0.74 & 2.4005 & 2.2 & 1.88 & 1.170213 & 0.588358 & 13 \\
\hline$\left[\mathrm{NiCl}_{4}\right] \mathrm{ClCs}_{3}{ }^{a}$ & $\mathrm{I} 4 / \mathrm{mcm}$ & 0.69 & 2.041241 & 1.81 & 1.88 & 0.962766 & 0.553182 & 14 \\
\hline$\left[\mathrm{CoCl}_{4}\right] \mathrm{ClTl}_{3}{ }^{a}$ & $\mathrm{I} 4 / \mathrm{mcm}$ & 0.65 & 2.008582 & 1.81 & 1.7 & 1.064706 & 0.572245 & 9 \\
\hline$\left[\mathrm{CoCl}_{4}\right] \mathrm{ClCs}_{3}{ }^{a}$ & $\mathrm{I} 4 / \mathrm{mcm}$ & 0.65 & 2.008582 & 1.81 & 1.88 & 0.962766 & 0.544331 & 9 \\
\hline$\left[\mathrm{CoBr}_{4}\right] \mathrm{BrCs}_{3}{ }^{a}$ & $\mathrm{I} 4 / \mathrm{mcm}$ & 0.65 & 2.131056 & 1.96 & 1.88 & 1.042553 & 0.554963 & 9 \\
\hline$\left[\mathrm{CoI}_{4}\right] \mathrm{ICs}_{3}{ }^{a}$ & Pnma & 0.65 & 2.327015 & 2.2 & 1.88 & 1.170213 & 0.570347 & 15 \\
\hline
\end{tabular}

References: $(a)$ the ionic radii $\left(r_{M}, r_{A}, r_{B}\right)$ are from the Materials Project Database (2020). 
Table S2 Formability, octahedral factor and golden ratio factor of 165 binary halide systems.

\begin{tabular}{|c|c|c|c|c|}
\hline No. & Systems & Formability & $\mu$ & $g$ \\
\hline \multicolumn{5}{|c|}{ Chloride } \\
\hline 1 & $\mathrm{NiCl} 2-3 \mathrm{LiCl}$ & No & 2.38158 & 0.787 \\
\hline 2 & $\mathrm{NiCl} 2-3 \mathrm{NaCl}$ & No & 1.30216 & 0.6321 \\
\hline 3 & $\mathrm{NiCl} 2-3 \mathrm{KCl}$ & Yes & 1.10366 & 0.5863 \\
\hline 4 & $\mathrm{NiCl} 2-3 \mathrm{RbCl}$ & Yes & 1.05233 & 0.573 \\
\hline 5 & $\mathrm{NiCl} 2-3 \mathrm{CsCl}$ & Yes & 0.96277 & 0.5482 \\
\hline 6 & $\mathrm{CuCl} 2-3 \mathrm{LiCl}$ & No & 2.38158 & 0.7996 \\
\hline 7 & $\mathrm{CuCl} 2-3 \mathrm{NaCl}$ & No & 1.30216 & 0.6422 \\
\hline 8 & $\mathrm{CuCl} 2-3 \mathrm{KCl}$ & Yes & 1.10366 & 0.5957 \\
\hline 9 & $\mathrm{CuCl} 2-3 \mathrm{RbCl}$ & Yes & 1.05233 & 0.5822 \\
\hline 10 & $\mathrm{CuCl} 2-3 \mathrm{CsCl}$ & Yes & 0.96277 & 0.5569 \\
\hline 11 & $\mathrm{ZnCl} 2-3 \mathrm{LiCl}$ & No & 2.38158 & 0.8028 \\
\hline 12 & $\mathrm{ZnCl} 2-3 \mathrm{NaCl}$ & No & 1.30216 & 0.6447 \\
\hline 13 & $\mathrm{ZnCl} 2-3 \mathrm{KCl}$ & Yes & 1.10366 & 0.598 \\
\hline 14 & $\mathrm{ZnCl} 2-3 \mathrm{RbCl}$ & Yes & 1.05233 & 0.5844 \\
\hline 15 & $\mathrm{ZnCl} 2-3 \mathrm{CsCl}$ & Yes & 0.96277 & 0.5591 \\
\hline 16 & $\mathrm{CoCl} 2-3 \mathrm{LiCl}$ & No & 2.38158 & 0.8044 \\
\hline 17 & $\mathrm{CoCl} 2-3 \mathrm{NaCl}$ & No & 1.30216 & 0.646 \\
\hline 18 & $\mathrm{CoCl} 2-3 \mathrm{KCl}$ & Yes & 1.10366 & 0.5992 \\
\hline 19 & $\mathrm{CoCl} 2-3 \mathrm{RbCl}$ & Yes & 1.05233 & 0.5856 \\
\hline 20 & $\mathrm{CoCl} 2-3 \mathrm{CsCl}$ & Yes & 0.96277 & 0.5602 \\
\hline 21 & $\mathrm{FeCl} 2-3 \mathrm{LiCl}$ & No & 2.38158 & 0.8154 \\
\hline 22 & $\mathrm{FeCl} 2-3 \mathrm{NaCl}$ & No & 1.30216 & 0.6548 \\
\hline 23 & $\mathrm{FeCl} 2-3 \mathrm{KCl}$ & Yes & 1.10366 & 0.6074 \\
\hline 24 & $\mathrm{FeCl} 2-3 \mathrm{RbCl}$ & Yes & 1.05233 & 0.5936 \\
\hline 25 & $\mathrm{FeCl} 2-3 \mathrm{CsCl}$ & Yes & 0.96277 & 0.5679 \\
\hline 26 & $\mathrm{MnCl} 2-3 \mathrm{LiCl}$ & No & 2.38158 & 0.8311 \\
\hline
\end{tabular}




\begin{tabular}{|c|c|c|c|c|}
\hline 27 & $\mathrm{MnCl} 2-3 \mathrm{NaCl}$ & No & 1.30216 & 0.6675 \\
\hline 28 & $\mathrm{MnCl} 2-3 \mathrm{KCl}$ & Yes & 1.10366 & 0.6191 \\
\hline 29 & $\mathrm{MnCl} 2-3 \mathrm{RbCl}$ & Yes & 1.05233 & 0.6051 \\
\hline 30 & $\mathrm{MnCl} 2-3 \mathrm{CsCl}$ & Yes & 0.96277 & 0.5788 \\
\hline 31 & $\mathrm{PdCl} 2-3 \mathrm{LiCl}$ & No & 2.38158 & 0.8405 \\
\hline 32 & $\mathrm{PdCl} 2-3 \mathrm{NaCl}$ & No & 1.30216 & 0.6751 \\
\hline 33 & $\mathrm{PdCl} 2-3 \mathrm{KCl}$ & Yes & 1.10366 & 0.6261 \\
\hline 34 & $\mathrm{PdCl} 2-3 \mathrm{RbCl}$ & Yes & 1.05233 & 0.612 \\
\hline 35 & $\mathrm{PdCl} 2-3 \mathrm{CsCl}$ & Yes & 0.96277 & 0.5854 \\
\hline 36 & $\mathrm{CdCl} 2-3 \mathrm{LiCl}$ & No & 2.38158 & 0.8689 \\
\hline 37 & $\mathrm{CdCl} 2-3 \mathrm{NaCl}$ & No & 1.30216 & 0.6978 \\
\hline 38 & $\mathrm{CdCl} 2-3 \mathrm{KCl}$ & No & 1.10366 & 0.6472 \\
\hline 39 & $\mathrm{CdCl} 2-3 \mathrm{RbCl}$ & Yes & 1.05233 & 0.6326 \\
\hline 40 & $\mathrm{CdCl} 2-3 \mathrm{CsCl}$ & Yes & 0.96277 & 0.6051 \\
\hline 41 & $\mathrm{HgCl} 2-3 \mathrm{LiCl}$ & No & 2.38158 & 0.8909 \\
\hline 42 & $\mathrm{HgCl} 2-3 \mathrm{NaCl}$ & No & 1.30216 & 0.7155 \\
\hline 43 & $\mathrm{HgCl} 2-3 \mathrm{KCl}$ & No & 1.10366 & 0.6637 \\
\hline 44 & $\mathrm{HgCl} 2-3 \mathrm{RbCl}$ & No & 1.05233 & 0.6486 \\
\hline 45 & $\mathrm{HgCl} 2-3 \mathrm{CsCl}$ & Yes & 0.96277 & 0.6205 \\
\hline 46 & $\mathrm{PbCl} 2-3 \mathrm{LiCl}$ & No & 2.38158 & 0.9444 \\
\hline 47 & $\mathrm{PbCl} 2-3 \mathrm{NaCl}$ & No & 1.30216 & 0.7585 \\
\hline 48 & $\mathrm{PbCl} 2-3 \mathrm{KCl}$ & No & 1.10366 & 0.7035 \\
\hline 49 & $\mathrm{PbCl} 2-3 \mathrm{RbCl}$ & No & 1.05233 & 0.6876 \\
\hline 50 & $\mathrm{PbCl} 2-3 \mathrm{CsCl}$ & No & 0.96277 & 0.6578 \\
\hline 51 & $\mathrm{PtCl} 2-3 \mathrm{LiCl}$ & No & 2.38158 & 0.7587 \\
\hline 52 & $\mathrm{PtCl} 2-3 \mathrm{NaCl}$ & No & 1.30216 & 0.6093 \\
\hline 53 & $\mathrm{PtCl} 2-3 \mathrm{KCl}$ & Yes & 1.10366 & 0.5652 \\
\hline 54 & $\mathrm{PtCl} 2-3 \mathrm{RbCl}$ & Yes & 1.05233 & 0.5524 \\
\hline 55 & $\mathrm{PtCl} 2-3 \mathrm{CsCl}$ & No & 0.96277 & 0.5284 \\
\hline
\end{tabular}




\begin{tabular}{|c|c|c|c|c|}
\hline \multicolumn{5}{|c|}{ Bromide } \\
\hline 56 & $\mathrm{NiBr} 2-3 \mathrm{LiBr}$ & No & 2.57895 & 0.7882 \\
\hline 57 & $\mathrm{NiBr} 2-3 \mathrm{NaBr}$ & No & 1.41007 & 0.64 \\
\hline 58 & $\mathrm{NiBr} 2-3 \mathrm{KBr}$ & No & 1.19512 & 0.5956 \\
\hline 59 & $\mathrm{NiBr} 2-3 \mathrm{RbBr}$ & Yes & 1.13953 & 0.5826 \\
\hline 60 & $\mathrm{NiBr} 2-3 \mathrm{CsBr}$ & Yes & 1.04255 & 0.5583 \\
\hline 61 & $\mathrm{CuBr} 2-3 \mathrm{LiBr}$ & No & 2.57895 & 0.8001 \\
\hline 62 & $\mathrm{CuBr} 2-3 \mathrm{NaBr}$ & No & 1.41007 & 0.6497 \\
\hline 63 & $\mathrm{CuBr} 2-3 \mathrm{KBr}$ & No & 1.19512 & 0.6046 \\
\hline 64 & $\mathrm{CuBr} 2-3 \mathrm{RbBr}$ & Yes & 1.13953 & 0.5914 \\
\hline 65 & $\mathrm{CuBr} 2-3 \mathrm{CsBr}$ & Yes & 1.04255 & 0.5668 \\
\hline 66 & $\mathrm{ZnBr} 2-3 \mathrm{LiBr}$ & No & 2.57895 & 0.8031 \\
\hline 67 & $\mathrm{ZnBr} 2-3 \mathrm{NaBr}$ & No & 1.41007 & 0.6521 \\
\hline 68 & $\mathrm{ZnBr} 2-3 \mathrm{KBr}$ & No & 1.19512 & 0.6068 \\
\hline 69 & $\mathrm{ZnBr} 2-3 \mathrm{RbBr}$ & Yes & 1.13953 & 0.5936 \\
\hline 70 & $\mathrm{ZnBr} 2-3 \mathrm{CsBr}$ & Yes & 1.04255 & 0.5689 \\
\hline 71 & $\mathrm{CoBr} 2-3 \mathrm{LiBr}$ & No & 2.57895 & 0.8046 \\
\hline 72 & $\mathrm{CoBr} 2-3 \mathrm{NaBr}$ & No & 1.41007 & 0.6533 \\
\hline 73 & $\mathrm{CoBr} 2-3 \mathrm{KBr}$ & No & 1.19512 & 0.6079 \\
\hline 74 & $\mathrm{CoBr} 2-3 \mathrm{RbBr}$ & Yes & 1.13953 & 0.5947 \\
\hline 75 & $\mathrm{CoBr} 2-3 \mathrm{CsBr}$ & Yes & 1.04255 & 0.5699 \\
\hline 76 & $\mathrm{FeBr} 2-3 \mathrm{LiBr}$ & No & 2.57895 & 0.815 \\
\hline 77 & $\mathrm{FeBr} 2-3 \mathrm{NaBr}$ & No & 1.41007 & 0.6617 \\
\hline 78 & $\mathrm{FeBr} 2-3 \mathrm{KBr}$ & No & 1.19512 & 0.6158 \\
\hline 79 & $\mathrm{FeBr} 2-3 \mathrm{RbBr}$ & Yes & 1.13953 & 0.6024 \\
\hline 80 & $\mathrm{FeBr} 2-3 \mathrm{CsBr}$ & Yes & 1.04255 & 0.5773 \\
\hline 81 & $\mathrm{MnBr} 2-3 \mathrm{LiBr}$ & No & 2.57895 & 0.8299 \\
\hline 82 & $\mathrm{MnBr} 2-3 \mathrm{NaBr}$ & No & 1.41007 & 0.6738 \\
\hline 83 & $\mathrm{MnBr} 2-3 \mathrm{KBr}$ & No & 1.19512 & 0.627 \\
\hline 84 & $\mathrm{MnBr} 2-3 \mathrm{RbBr}$ & Yes & 1.13953 & 0.6134 \\
\hline
\end{tabular}




\begin{tabular}{|c|c|c|c|c|}
\hline 85 & $\mathrm{MnBr} 2-3 \mathrm{CsBr}$ & Yes & 1.04255 & 0.5878 \\
\hline 86 & PdBr2-3LiBr & No & 2.57895 & 0.8388 \\
\hline 87 & $\mathrm{PdBr} 2-3 \mathrm{NaBr}$ & No & 1.41007 & 0.6811 \\
\hline 88 & $\mathrm{PdBr} 2-3 \mathrm{KBr}$ & No & 1.19512 & 0.6338 \\
\hline 89 & $\mathrm{PdBr} 2-3 \mathrm{RbBr}$ & Yes & 1.13953 & 0.62 \\
\hline 90 & $\mathrm{PdBr} 2-3 \mathrm{CsBr}$ & Yes & 1.04255 & 0.5942 \\
\hline 91 & $\mathrm{CdBr} 2-3 \mathrm{LiBr}$ & No & 2.57895 & 0.8656 \\
\hline 92 & $\mathrm{CdBr} 2-3 \mathrm{NaBr}$ & No & 1.41007 & 0.7028 \\
\hline 93 & $\mathrm{CdBr} 2-3 \mathrm{KBr}$ & No & 1.19512 & 0.654 \\
\hline 94 & $\mathrm{CdBr} 2-3 \mathrm{RbBr}$ & Yes & 1.13953 & 0.6398 \\
\hline 95 & $\mathrm{CdBr} 2-3 \mathrm{CsBr}$ & Yes & 1.04255 & 0.6131 \\
\hline 96 & $\mathrm{HgBr} 2-3 \mathrm{LiBr}$ & No & 2.57895 & 0.8864 \\
\hline 97 & $\mathrm{HgBr} 2-3 \mathrm{NaBr}$ & No & 1.41007 & 0.7197 \\
\hline 98 & $\mathrm{HgBr} 2-3 \mathrm{KBr}$ & No & 1.19512 & 0.6697 \\
\hline 99 & $\mathrm{HgBr} 2-3 \mathrm{RbBr}$ & No & 1.13953 & 0.6552 \\
\hline 100 & $\mathrm{HgBr} 2-3 \mathrm{CsBr}$ & Yes & 1.04255 & 0.6279 \\
\hline 101 & $\mathrm{PbBr} 2-3 \mathrm{LiBr}$ & No & 2.57895 & 0.9369 \\
\hline 102 & $\mathrm{PbBr} 2-3 \mathrm{NaBr}$ & No & 1.41007 & 0.7607 \\
\hline 103 & $\mathrm{PbBr} 2-3 \mathrm{KBr}$ & No & 1.19512 & 0.7079 \\
\hline 104 & $\mathrm{PbBr} 2-3 \mathrm{RbBr}$ & No & 1.13953 & 0.6925 \\
\hline 105 & $\mathrm{PbBr} 2-3 \mathrm{CsBr}$ & No & 1.04255 & 0.6637 \\
\hline 106 & $\mathrm{PtBr} 2-3 \mathrm{LiBr}$ & No & 2.57895 & 0.7615 \\
\hline 107 & $\mathrm{PtBr} 2-3 \mathrm{NaBr}$ & No & 1.41007 & 0.6183 \\
\hline 108 & $\mathrm{PtBr} 2-3 \mathrm{KBr}$ & No & 1.19512 & 0.5753 \\
\hline 109 & PtBr2-3RbBr & Yes & 1.13953 & 0.5628 \\
\hline 110 & $\mathrm{PtBr} 2-3 \mathrm{CsBr}$ & No & 1.04255 & 0.5394 \\
\hline \multicolumn{5}{|c|}{ Iodide } \\
\hline 111 & NiI2-3LiI & No & 2.89474 & 0.7899 \\
\hline 112 & $\mathrm{NiI} 2-3 \mathrm{NaI}$ & No & 1.58273 & 0.6513 \\
\hline
\end{tabular}




\begin{tabular}{|c|c|c|c|c|}
\hline 113 & NiI2-3KI & No & 1.34146 & 0.6089 \\
\hline 114 & NiI2-3RbI & No & 1.27907 & 0.5965 \\
\hline 115 & NiI2-3CsI & Yes & 1.17021 & 0.5731 \\
\hline 116 & CuI2-3LiI & No & 2.89474 & 0.8009 \\
\hline 117 & $\mathrm{CuI} 2-3 \mathrm{NaI}$ & No & 1.58273 & 0.6603 \\
\hline 118 & CuI2-3KI & No & 1.34146 & 0.6173 \\
\hline 119 & $\mathrm{CuI} 2-3 \mathrm{RbI}$ & No & 1.27907 & 0.6047 \\
\hline 120 & CuI2-3CsI & Yes & 1.17021 & 0.581 \\
\hline 121 & ZnI2-3LiI & No & 2.89474 & 0.8036 \\
\hline 122 & $\mathrm{ZnI} 2-3 \mathrm{NaI}$ & No & 1.58273 & 0.6626 \\
\hline 123 & ZnI2-3KI & No & 1.34146 & 0.6194 \\
\hline 124 & $\mathrm{ZnI2-3RbI}$ & No & 1.27907 & 0.6068 \\
\hline 125 & ZnI2-3CsI & Yes & 1.17021 & 0.583 \\
\hline 126 & CoI2-3LiI & No & 2.89474 & 0.805 \\
\hline 127 & $\mathrm{CoI} 2-3 \mathrm{NaI}$ & No & 1.58273 & 0.6637 \\
\hline 128 & CoI2-3KI & No & 1.34146 & 0.6205 \\
\hline 129 & $\mathrm{CoI} 2-3 \mathrm{RbI}$ & No & 1.27907 & 0.6078 \\
\hline 130 & CoI2-3CsI & Yes & 1.17021 & 0.584 \\
\hline 131 & FeI2-3LiI & No & 2.89474 & 0.8145 \\
\hline 132 & FeI2-3NaI & No & 1.58273 & 0.6716 \\
\hline 133 & FeI2-3KI & No & 1.34146 & 0.6279 \\
\hline 134 & FeI2-3RbI & No & 1.27907 & 0.6151 \\
\hline 135 & FeI2-3CsI & Yes & 1.17021 & 0.5909 \\
\hline 136 & MnI2-3LiI & No & 2.89474 & 0.8282 \\
\hline 137 & $\mathrm{MnI} 2-3 \mathrm{NaI}$ & No & 1.58273 & 0.6829 \\
\hline 138 & MnI2-3KI & No & 1.34146 & 0.6384 \\
\hline 139 & MnI2-3RbI & No & 1.27907 & 0.6254 \\
\hline 140 & MnI2-3CsI & Yes & 1.17021 & 0.6009 \\
\hline 141 & PdI2-3LiI & No & 2.89474 & 0.8364 \\
\hline 142 & PdI2-3NaI & No & 1.58273 & 0.6896 \\
\hline
\end{tabular}




\begin{tabular}{|c|c|c|c|c|}
\hline 143 & PdI2-3KI & No & 1.34146 & 0.6447 \\
\hline 144 & PdI2-3RbI & No & 1.27907 & 0.6316 \\
\hline 145 & PdI2-3CsI & Yes & 1.17021 & 0.6068 \\
\hline 146 & CdI2-3LiI & No & 2.89474 & 0.861 \\
\hline 147 & $\mathrm{CdI} 2-3 \mathrm{NaI}$ & No & 1.58273 & 0.7099 \\
\hline 148 & CdI2-3KI & No & 1.34146 & 0.6637 \\
\hline 149 & $\mathrm{CdI} 2-3 \mathrm{RbI}$ & No & 1.27907 & 0.6501 \\
\hline 150 & CdI2-3CsI & Yes & 1.17021 & 0.6246 \\
\hline 151 & HgI2-3LiI & No & 2.89474 & 0.8801 \\
\hline 152 & $\mathrm{HgI} 2-3 \mathrm{NaI}$ & No & 1.58273 & 0.7257 \\
\hline 153 & $\mathrm{HgI} 2-3 \mathrm{KI}$ & No & 1.34146 & 0.6784 \\
\hline 154 & HgI2-3RbI & No & 1.27907 & 0.6646 \\
\hline 155 & HgI2-3CsI & Yes & 1.17021 & 0.6385 \\
\hline 156 & $\mathrm{PbI} 2-3 \mathrm{LiI}$ & No & 2.89474 & 0.9266 \\
\hline 157 & $\mathrm{PbI} 2-3 \mathrm{NaI}$ & No & 1.58273 & 0.764 \\
\hline 158 & $\mathrm{PbI} 2-3 \mathrm{KI}$ & No & 1.34146 & 0.7142 \\
\hline 159 & $\mathrm{PbI} 2-3 \mathrm{RbI}$ & No & 1.27907 & 0.6997 \\
\hline 160 & $\mathrm{PbI} 2-3 \mathrm{CsI}$ & No & 1.17021 & 0.6722 \\
\hline 161 & PtI2-3LiI & No & 2.89474 & 0.7653 \\
\hline 162 & PtI2-3NaI & No & 1.58273 & 0.631 \\
\hline 163 & PtI2-3KI & No & 1.34146 & 0.5899 \\
\hline 164 & PtI2-3RbI & No & 1.27907 & 0.5779 \\
\hline 165 & PtI2-3CsI & Yes & 1.17021 & 0.5552 \\
\hline
\end{tabular}


Table S3. Crystallography and refinement details.

\begin{tabular}{|c|c|c|c|c|c|c|}
\hline & 1 & 2 & 3 & 4 & 5 & 6 \\
\hline Empirical formula & {$\left[\mathrm{MnCl}_{4}\right] \mathrm{ClCs}_{3}$} & {$\left[\mathrm{MnCl}_{3} \mathrm{Br}\right] \mathrm{BrCs}_{3}$} & $\begin{array}{l}{\left[\mathrm{MnCl}_{2} \mathrm{Br}_{2}\right] \mathrm{BrCs}} \\
3\end{array}$ & {$\left[\mathrm{MnBr}_{4}\right] \mathrm{BrCs}_{3}$} & {$\left[\mathrm{MnBr}_{2} \mathrm{I}_{2}\right] \mathrm{ICs}_{3}$} & $\begin{array}{l}\mathrm{CsMn}_{0.33} \mathrm{Br}_{0.67} \mathrm{I} \\
\left(\left[\mathrm{MnBr}_{2} \mathrm{I}_{2}\right] \mathrm{ICs}_{3}\right)\end{array}$ \\
\hline Formula weight & 630.92 & 719.84 & 764.30 & 853.22 & 905.27 & 331.40 \\
\hline Crystal size, $\mathrm{mm}$ & $\begin{array}{l}0.560 \times 0.410 \times 0 . \\
360\end{array}$ & $\begin{array}{l}0.300 \times 0.270 \times 0 . \\
120\end{array}$ & $\begin{array}{l}0.260 \times 0.240 \times 0 . \\
200\end{array}$ & $\begin{array}{l}0.130 \times 0.100 \times 0 . \\
040\end{array}$ & $\begin{array}{l}0.220 \times 0.180 \times 0 \\
160\end{array}$ & $\begin{array}{l}0.260 \times 0.170 \times 0 . \\
090\end{array}$ \\
\hline Crystal system & Tetragonal & Tetragonal & Tetragonal & Tetragonal & Tetragonal & Tetragonal \\
\hline Space group & $\mathrm{I} 4 / \mathrm{mcm}$ & $\mathrm{I} 4 / \mathrm{mcm}$ & $\mathrm{I} 4 / \mathrm{mcm}$ & $\mathrm{I} 4 / \mathrm{mcm}$ & $\mathrm{P} 4212$ & $\mathrm{I} 4 / \mathrm{mcm}$ \\
\hline$a, \AA$ & $9.1751(3)$ & $9.4660(11)$ & $9.4990(5)$ & $9.6044(6)$ & $14.4125(4)$ & $10.0942(5)$ \\
\hline$b, \AA$ & $9.1751(3)$ & $9.4660(11)$ & $9.4990(5)$ & $9.6044(6)$ & $14.4125(4)$ & $10.0942(5)$ \\
\hline$c, \AA$ & $14.8827(10)$ & $14.999(4)$ & $15.0890(13)$ & $15.5668(13)$ & $15.2106(7)$ & $15.8800(10)$ \\
\hline$\alpha, \operatorname{deg}$ & 90 & 90 & 90 & 90 & 90 & 90 \\
\hline$\beta, \operatorname{deg}$ & 90 & 90 & 90 & 90 & 90 & 90 \\
\hline$\gamma, \operatorname{deg}$ & 90 & 90 & 90 & 90 & 90 & 90 \\
\hline$V, \AA^{3}$ & $1252.86(12)$ & $1344.0(5)$ & $1361.50(19)$ & $1436.0(2)$ & $3159.5(2)$ & $1618.06(19)$ \\
\hline$Z$ & 4 & 4 & 4 & 4 & 8 & 12 \\
\hline $\mathrm{D}_{\text {cakd }} \mathrm{mg} \mathrm{m}^{-3}$ & 3.345 & 3.558 & 3.729 & 3.947 & 3.806 & 4.081 \\
\hline $\mathrm{F}_{000}$ & 1100 & 1244 & 1316 & 1460 & 3064 & 1676 \\
\hline Temperature, $\mathrm{K}$ & $298(2)$ & $298(2)$ & $293(2)$ & $297(2)$ & $296(2)$ & $293(2)$ \\
\hline Wavelength, $\AA$ & 0.71073 & 0.71073 & 0.71073 & 1.34138 & 0.71073 & 0.71073 \\
\hline $\begin{array}{l}\text { Absorption } \\
\text { coefficient, } \mathrm{mm}^{-1}\end{array}$ & 10.648 & 15.475 & 18.014 & 56.061 & 13.801 & 18.083 \\
\hline $\begin{array}{ll}2 \theta_{\max }, & \operatorname{deg} \\
\text { (Completeness) }\end{array}$ & $50.484(100 \%)$ & $50.484(100 \%)$ & $50.484(100 \%)$ & $53.594(100 \%)$ & $50.484(99.8 \%)$ & $50.484(99.8 \%)$ \\
\hline $\begin{array}{l}\text { no. of collected } \\
\text { reflections }\end{array}$ & 9689 & 27981 & 15835 & 4796 & 23943 & 16612 \\
\hline no. of unique ref. $\left(\mathrm{R}_{\text {int }}\right)$ & $362(0.1350)$ & $405(0.1236)$ & $535(0.0988)$ & $451(0.0602)$ & $3512(0.0771)$ & $438(0.0735)$ \\
\hline $\begin{array}{l}\text { Data/restraints/param } \\
\text { eters }\end{array}$ & $362 / 0 / 17$ & $405 / 0 / 18$ & $535 / 0 / 18$ & $451 / 0 / 17$ & $3512 / 114 / 154$ & $438 / 0 / 18$ \\
\hline$R_{1}, \mathrm{w} R_{2}[\mathrm{obs} I>2 \sigma(I)]$ & $0.1189,0.3027$ & $0.0267,0.0671$ & $0.0275,0.0565$ & $0.0401,0.1046$ & $0.1385,0.3953$ & $0.0227,0.0511$ \\
\hline$R_{1}, \mathrm{w} R_{2}$ (all data) & $0.1189,0.3042$ & $0.0278,0.0677$ & $0.0351,0.0588$ & $0.0404,0.1048$ & $0.1546,0.4101$ & $0.0251,0.0520$ \\
\hline $\begin{array}{l}\text { Residual peak/hole, e. } \\
\AA^{-3}\end{array}$ & $6.189 /-5.467$ & $0.703 /-1.128$ & $0.776 /-0.757$ & $1.218 /-1.999$ & $5.125 /-2.360$ & $0.700 /-0.591$ \\
\hline $\begin{array}{l}\text { Goodness-of-fit on } \\
\mathrm{F}^{\wedge} 2\end{array}$ & 1.255 & 1.181 & 1.113 & 1.029 & 1.067 & 1.097 \\
\hline
\end{tabular}


Table S4. Crystal structure of anti-perovskites 1, 2, 4-6 unit cell towards plane (001) and along [110].

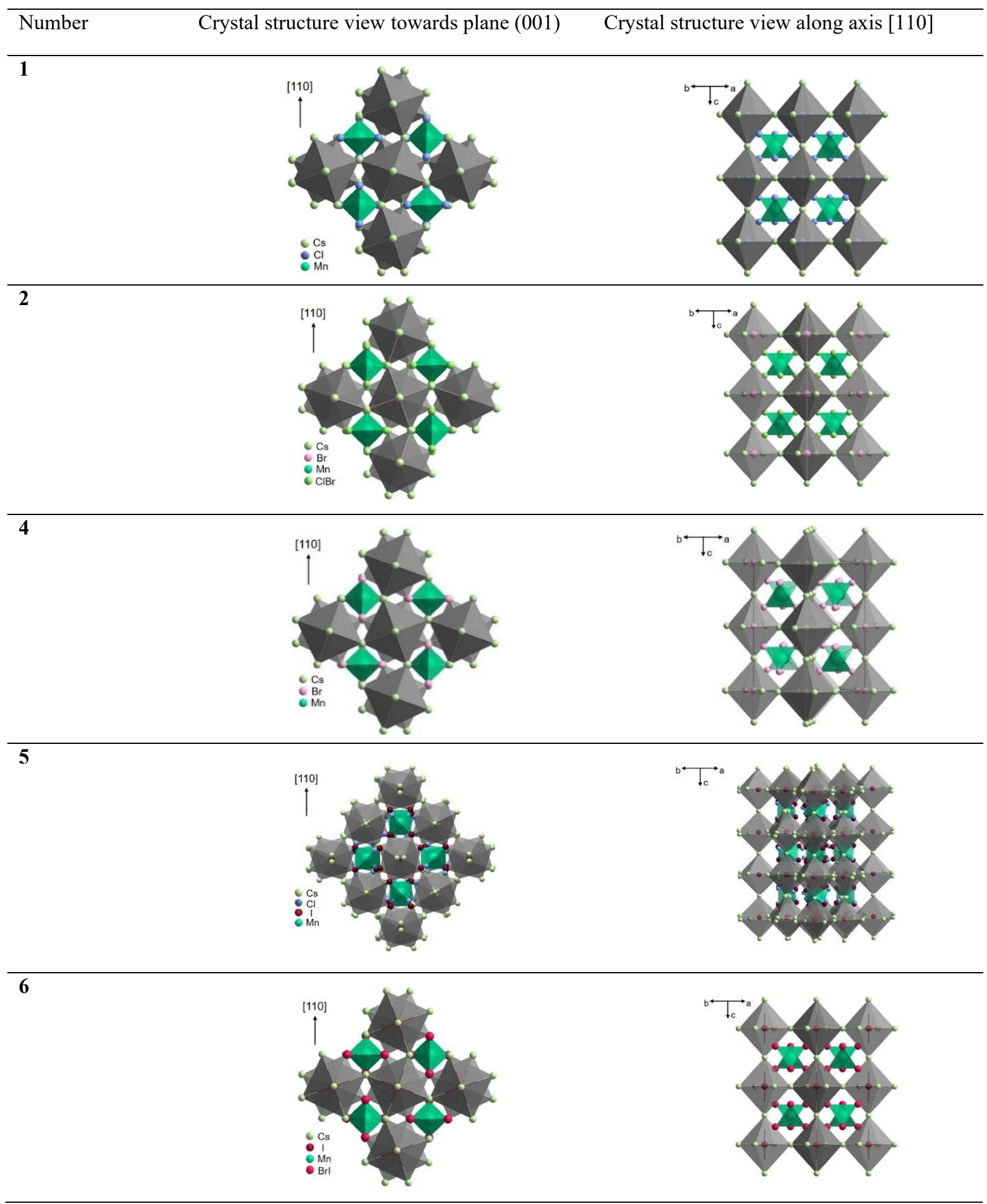


Table S5. Luminescent data of crystals 1-6.

\begin{tabular}{ccccc}
\hline No. & Crystal & PL peak (nm) & Lifetime (ms) & PLQY (\%, R.T.) \\
\hline $\mathbf{1}$ & {$\left[\mathrm{MnCl}_{4}\right] \mathrm{ClCs}_{3}$} & 517 & 0.208 & 81.3 \\
\hline $\mathbf{2}$ & {$\left[\mathrm{MnCl}_{3} \mathrm{Br}\right] \mathrm{BrCs}_{3}$} & 518 & 0.239 & 91.5 \\
\hline $\mathbf{3}$ & {$\left[\mathrm{MnCl}_{2} \mathrm{Br}_{2}\right] \mathrm{BrCs}_{3}$} & 520 & 0.249 & 93.5 \\
\hline $\mathbf{4}$ & {$\left[\mathrm{MnBr}_{4}\right] \mathrm{BrCs}_{3}$} & 522 & 0.290 & 89.1 \\
\hline $\mathbf{5}$ & {$\left[\mathrm{MnCl}_{2} \mathrm{I}_{2}\right] \mathrm{ICs}_{3}$} & 537 & 0.332 & 87.3 \\
\hline $\mathbf{6}$ & {$\left[\mathrm{MnBr}_{2} \mathrm{I}_{2}\right] \mathrm{ICs}_{3}$} & 541 & 0.350 & 86.2 \\
\hline
\end{tabular}

Table S6. Luminescent data of crystal $\mathbf{3}$ and corresponding thermal-evaporated film.

\begin{tabular}{cccc}
\hline & PL peak $(\mathbf{n m})$ & Lifetime $(\mathbf{m s})$ & PLQY (\%, R.T.) \\
\hline Crystal & 520 & 0.249 & 93.5 \\
\hline Film & 520 & 0.223 & 86.3 \\
\hline
\end{tabular}


Table S7. Photophysical properties of single crystal $\left[\mathrm{MnCl}_{2} \mathrm{Br}_{2}\right] \mathrm{BrCs}_{3}$ from $77 \mathrm{~K}$ to $523 \mathrm{~K}$.

\begin{tabular}{|c|c|c|c|c|}
\hline Temperature (K) & $\lambda_{\text {exc }}(\mathrm{nm})$ & $\lambda_{\mathrm{em}}(\mathrm{nm})$ & Stokes Shift (nm) & $\tau_{\mathrm{av}}(\mathrm{ms})$ \\
\hline 77 & 365 & 521 & 156 & 0.383 \\
\hline 90 & 365 & 520 & 155 & 0.361 \\
\hline 110 & 365 & 522 & 157 & 0.337 \\
\hline 130 & 365 & 521 & 156 & 0.310 \\
\hline 150 & 365 & 522 & 157 & 0.288 \\
\hline 150 & 365 & 520 & 155 & 0.288 \\
\hline 170 & 365 & 521 & 156 & 0.280 \\
\hline 190 & 365 & 521 & 156 & 0.270 \\
\hline 210 & 365 & 520 & 155 & 0.264 \\
\hline 230 & 365 & 520 & 155 & 0.263 \\
\hline 265 & 365 & 521 & 156 & 0.255 \\
\hline 298 & 365 & 522 & 157 & 0.249 \\
\hline 323 & 365 & 521 & 156 & 0.210 \\
\hline 348 & 365 & 520 & 155 & 0.208 \\
\hline 373 & 365 & 520 & 155 & 0.206 \\
\hline 398 & 365 & 520 & 155 & 0.186 \\
\hline 423 & 365 & 521 & 156 & 0.179 \\
\hline 448 & 365 & 520 & 155 & 0.163 \\
\hline 473 & 365 & 520 & 155 & 0.155 \\
\hline 498 & 365 & 521 & 156 & 0.147 \\
\hline 523 & 365 & 520 & 155 & 0.137 \\
\hline
\end{tabular}

Table S8. Electroluminescent data of device 1 (PMMA optimization) and device 2.

\begin{tabular}{|c|c|c|c|c|c|c|}
\hline & $\begin{array}{c}\text { EL peak } \\
(\mathbf{n m})\end{array}$ & $\begin{array}{c}\text { Peak current } \\
\text { density }\left(\mathrm{mA} \mathrm{cm}^{-2}\right)\end{array}$ & $\begin{array}{l}\operatorname{Lumi}_{\max } \\
\left(\mathbf{c d ~ m ^ { - 2 } )}\right)\end{array}$ & $\mathrm{EQE}_{\max }(\%)$ & $\mathrm{EQE}_{\text {avg }}(\%)$ & Lifetime (min) \\
\hline Device 1 & 520 & 69.5 & 3990 & 12.5 & 9.7 & $756\left(100 \mathrm{~cd} \mathrm{~m}^{-2}\right)$ \\
\hline Device 2 & 520 & 29.3 & 1490 & 6.0 & 5.0 & - \\
\hline
\end{tabular}




\section{Tanabe-Sugano energy level diagram}

In this work, the crystal field Dq and Racah B parameter are calculated by the equations (1)-(3) listed below: ${ }^{16}$

$$
\begin{aligned}
& { }^{6} \mathrm{~A}_{1}(6 \mathrm{~S}) \rightarrow{ }^{4} \mathrm{~A}_{1}{ }^{4} \mathrm{E}(\mathrm{G})=10 \mathrm{~B}+5 \mathrm{C} \\
& { }^{6} \mathrm{~A}_{1}(6 \mathrm{~S}) \rightarrow{ }^{4} \mathrm{E}(\mathrm{D})=17 \mathrm{~B}+5 \mathrm{C} \\
& { }^{6} \mathrm{~A}_{1}(6 \mathrm{~S}) \rightarrow{ }^{4} \mathrm{~T}_{2}(\mathrm{G})=-10 \mathrm{Dq}+18 \mathrm{~B}+6 \mathrm{C}-\left(26 \mathrm{~B}^{2} / 10 \mathrm{Dq}\right) \\
& 21929=10 \mathrm{~B}+5 \mathrm{C} \\
& 19607=-10 \mathrm{Dq}+18 \mathrm{~B}+6 \mathrm{C}-\left(26 \mathrm{~B}^{2} / 10 \mathrm{Dq}\right)
\end{aligned}
$$

The calculation values are $\mathrm{Dq}=819.87 \mathrm{~cm}^{-1}, \mathrm{~B}=762.57 \mathrm{~cm}^{-1}$, and $\mathrm{C}=3041.08 \mathrm{~cm}^{-1}$. The $\mathrm{Dq} / \mathrm{B}$ value can be calculated to be $\sim 1.08$. 


\section{DFT calculation}

The first-principles density functional calculations were performed using Perdew-BurkeErnzerhof $(\mathrm{PBE})^{17}$ functional within the spin-polarized GGA $+\mathrm{U}^{18}$ approximation as implemented in the Cambridge serial total energy package $(\mathrm{CASTEP})^{19}$ code. The Vanderbilt ultrasoft pseudopotential $^{20}$ was used with a cutoff energy of $370 \mathrm{eV}$. Geometric convergence tolerances were set for maximum force of $0.03 \mathrm{eV} / \AA$, maximum energy change of $10^{-5} \mathrm{eV} /$ atom, maximum displacement of $0.001 \AA$ and maximum stress of $0.5 \mathrm{GPa}$. Density mixing electronic minimisation was implemented and the self-consistent field (SCF) tolerance was set to "fine" with high accuracy of $10^{-6} \mathrm{eV} /$ atom for energy convergence. The Hubbard U correction was introduced to describe the effect of localized d electrons of Mn ions, which $\mathrm{U}$ value is set to $1.5 \mathrm{eV}$ to correct the calculated bandgap and better match with the experimental result. The relative quantities of the Br-4p, Mn-3d and Mn-3p orbitals, which form the VBM, were calculated from the ratio of the integral area of their spin-up states PDOS peaks that were closest to Fermi level on the valence band side. 

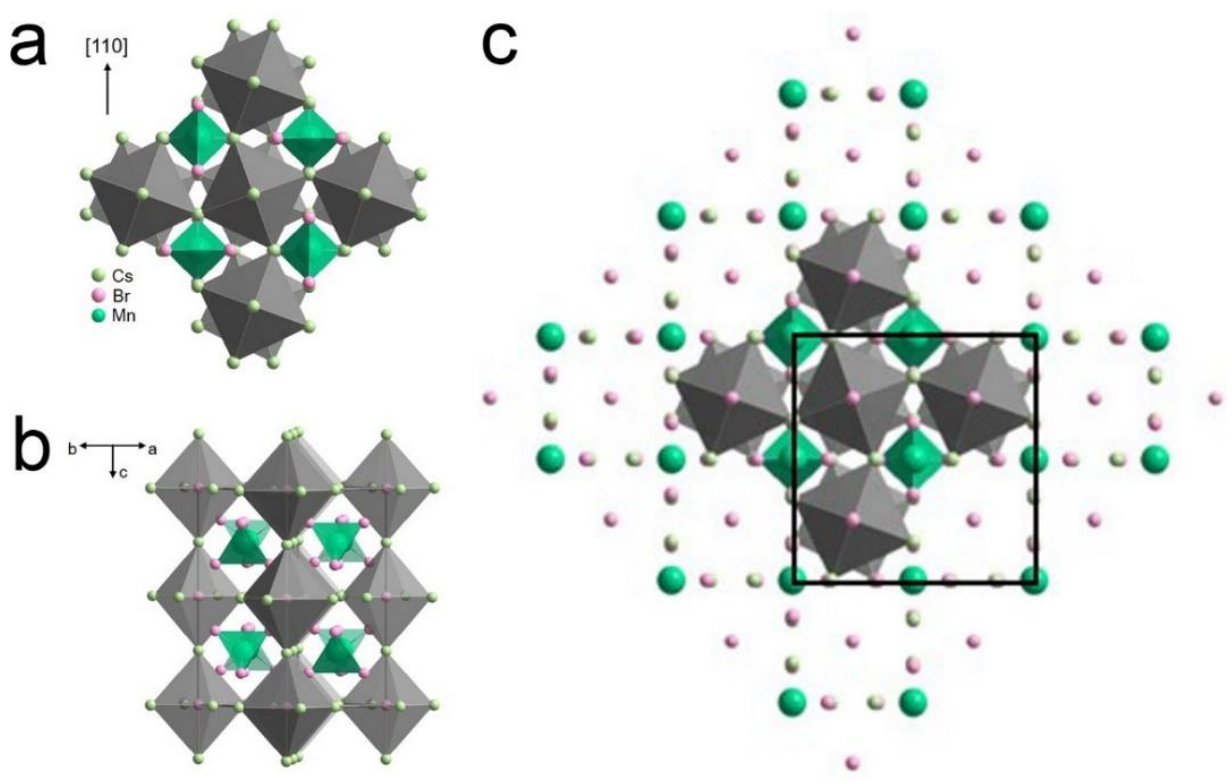

Figure S1. (a) Unit cell structure of crystal $\left[\mathrm{MnBr}_{4}\right] \mathrm{BrCs}_{3}$ towards plane (001). (greenish yellow: Cs; pink: Br; green: Mn) (b) Unit cell structure of crystal [ $\left.\mathrm{MnBr}_{4}\right] \mathrm{BrCs}_{3}$ along [110]. (c) Super cell $(3 \times 3 \times 3)$ structure of crystal $\left[\mathrm{MnBr}_{4}\right] \mathrm{BrCs}_{3}$. The extracted analysis unit is marked by the black rectangle.

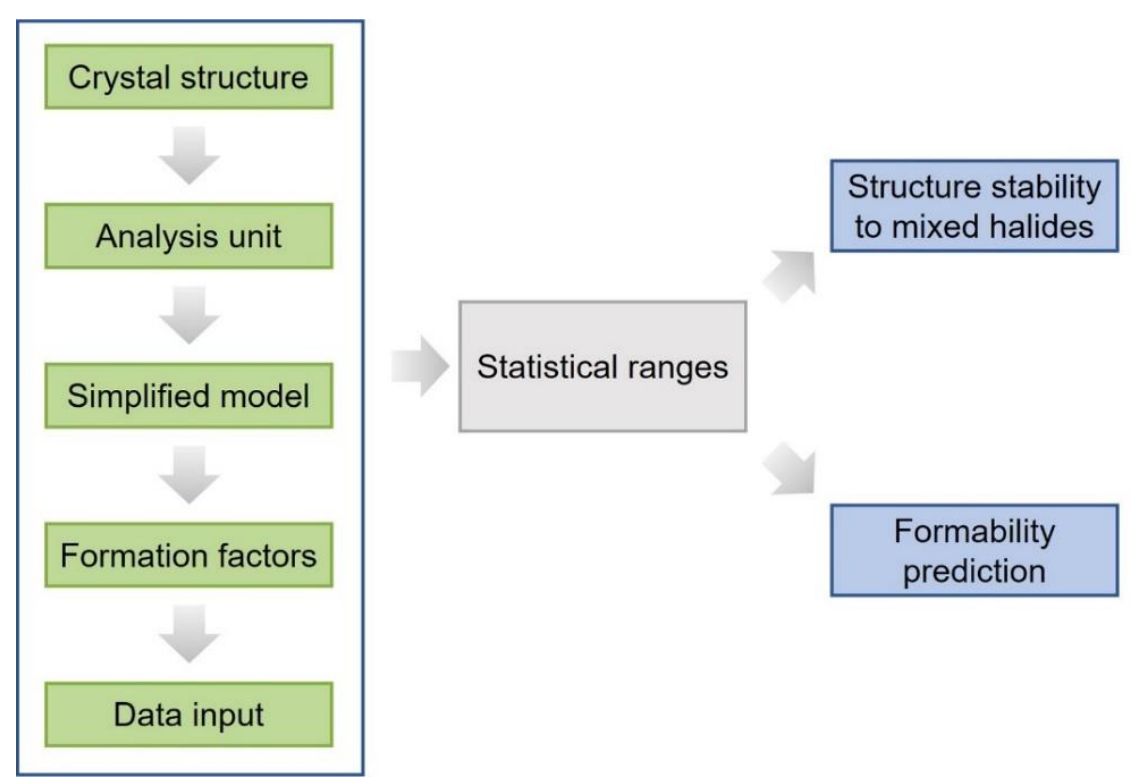

Figure S2. Overview of statistical analysis of $\left[\mathrm{MX}_{4}\right] \mathrm{XA}_{3}$-type anti-perovskite structure. 


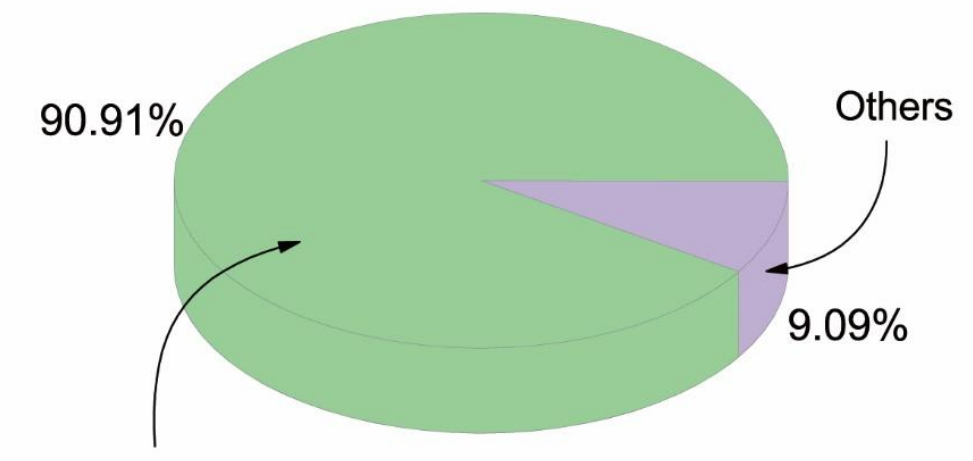

$\left[\mathrm{MX}_{4}\right]$ tetrahedra separated by 3D or 1D anti-perovskite structures

Figure S3. The accuracy analysis pie chart of $\mu-g$ structure map towards existing $\left[\mathrm{MX}_{4}\right] \mathrm{XA}_{3}$-type anti-perovskites.

In the 22 experimentally synthesized $\left[\mathrm{MX}_{4}\right] \mathrm{XA}_{3}$ materials, 13 compounds adopt in the tetrahedral space group with $\mathrm{I} / \mathrm{mcm}$ symmetry, whose structure can be regarded as $\left[\mathrm{MX}_{4}\right]$ tetrahedra separated by 3D XA 6 octahedral framework. 7 compounds adopt in the orthorhombic space group with Pnma and Pbca symmetry, whose structure can be regarded as the $\left[\mathrm{MX}_{4}\right]$ tetrahedra separated by $1 \mathrm{D} \mathrm{XA}_{6}$ octahedral chains. 2 compounds adopt in the tetragonal space group with $\mathrm{P} 4 / \mathrm{mcm}$ symmetry, in which $\left[\mathrm{MX}_{4}\right]$ forms a plane quadrangle instead of tetrahedron. Considering that $\left[\mathrm{MX}_{4}\right]$ tetrahedra separated by $\mathrm{XA}_{6}$ octahedra was our targeted structure, the last two compounds were not included when we set the ranges of $\mu$ and $g$. 


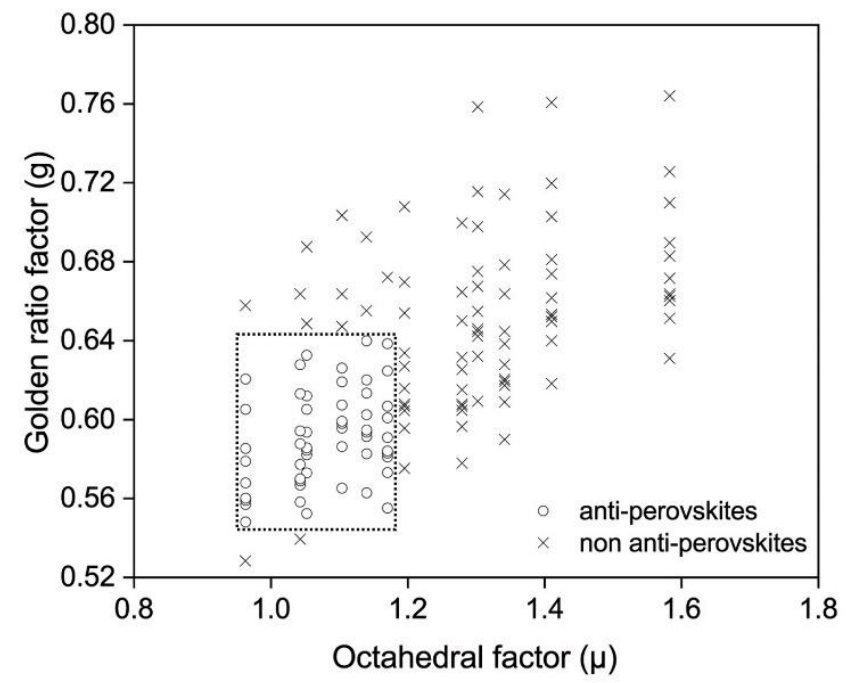

Figure S4. The $\mu-g$ structure map. The predicted $\left[\mathrm{MX}_{4}\right] \mathrm{XA}_{3}$-type anti-perovskites are included in the rectangle.

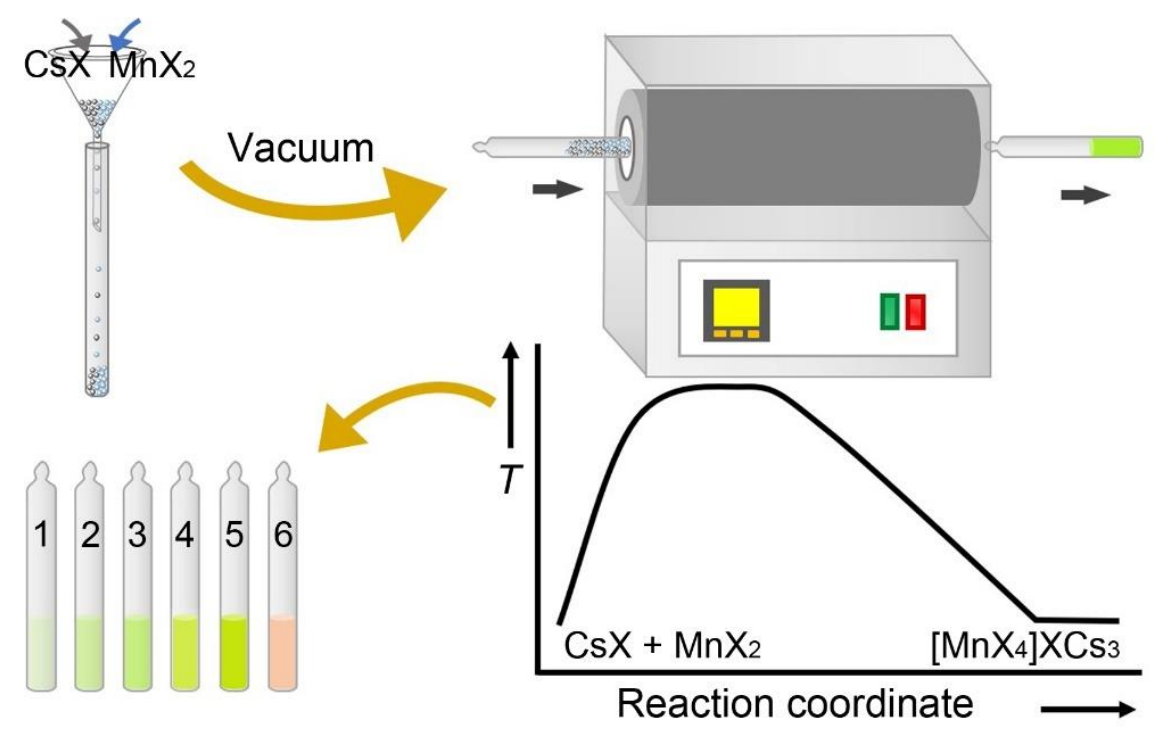

Figure S5. Schematic diagram of single crystals 1-6 synthesis by solid-state reaction method in vacuum. 

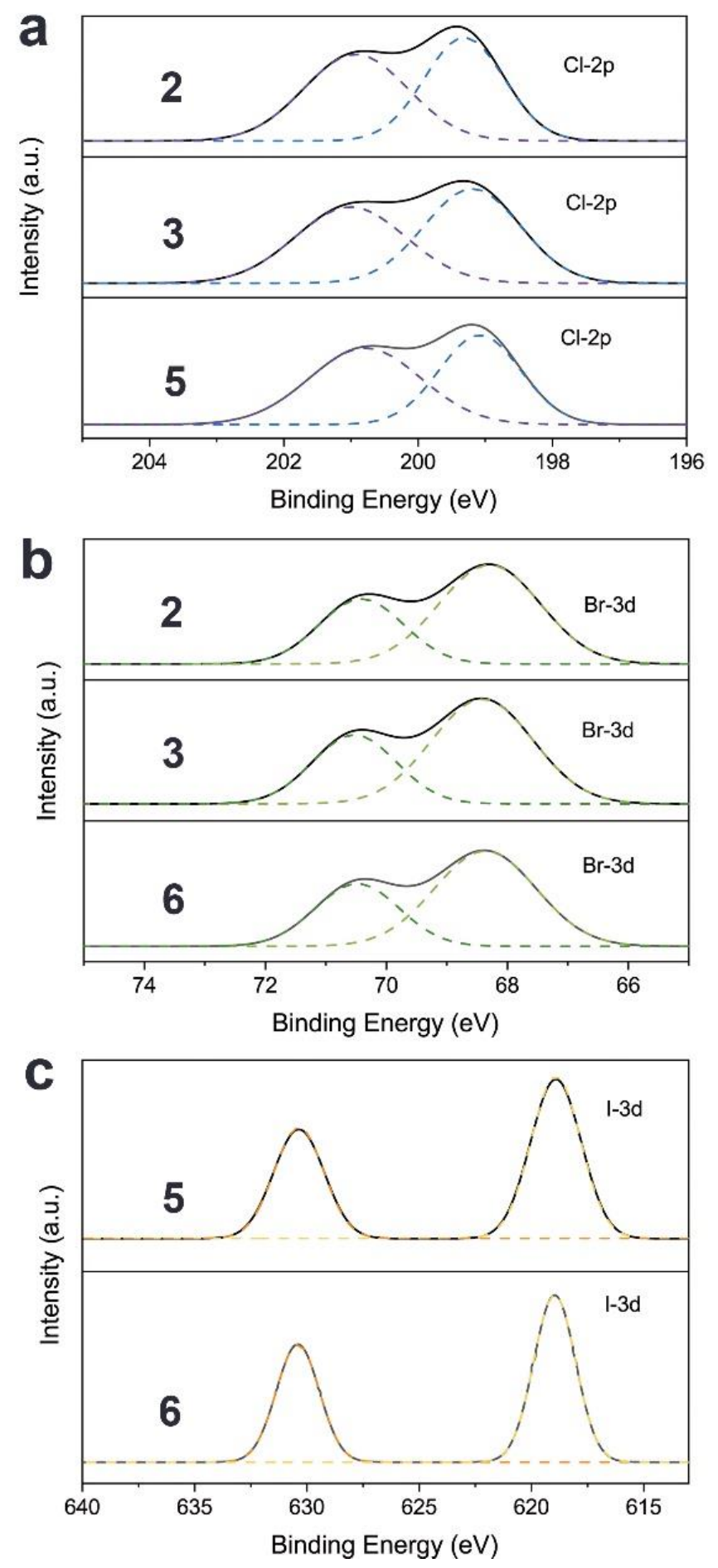

Figure S6. Photoelectron spectra from X-ray photoelectron spectroscopy (XPS) and Gaussian peak fitting for (a) Cl-2p, (b) Br-3d, and (c) I-3d core levels. 
Table S9. Halide ratios in the mixed halide systems.

\begin{tabular}{ccc}
\hline No. & Crystal & Halide ratio \\
\hline $\mathbf{2}$ & {$\left[\mathrm{MnCl}_{3} \mathrm{Br}\right] \mathrm{BrCs}_{3}$} & $\mathrm{Cl} / \mathrm{Br} \sim 1.51$ \\
\hline $\mathbf{3}$ & {$\left[\mathrm{MnCl}_{2} \mathrm{Br}_{2}\right] \mathrm{BrCs}_{3}$} & $\mathrm{Br} / \mathrm{Cl} \sim 1.50$ \\
\hline $\mathbf{5}$ & {$\left[\mathrm{MnCl}_{2} \mathrm{I}_{2}\right] \mathrm{ICs}_{3}$} & $\mathrm{I} / \mathrm{Cl} \sim 1.50$ \\
\hline $\mathbf{6}$ & {$\left[\mathrm{MnBr}_{2} \mathrm{I}_{2}\right] \mathrm{ICs}_{3}$} & $\mathrm{I} / \mathrm{Br} \sim 1.51$ \\
\hline
\end{tabular}



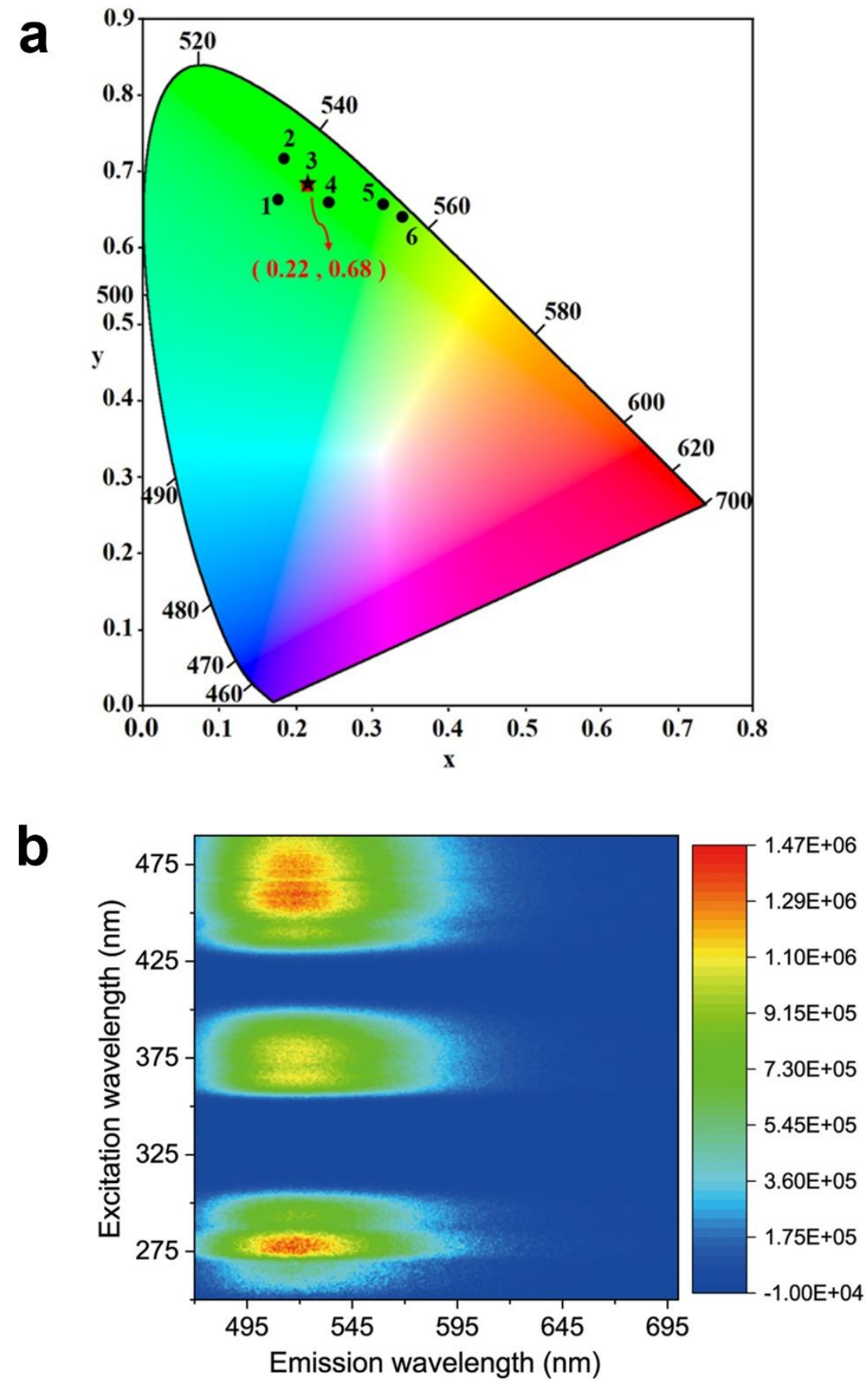

Figure S7. The high and intrinsic optical performance of crystal $\left[\mathrm{MnCl}_{2} \mathrm{Br}_{2}\right] \mathrm{BrCs}_{3}$. (a) Chromaticity of the emission extracted from the PL spectra. (b) 3D EEM fluorescence spectroscopy of single crystal $\left[\mathrm{MnCl}_{2} \mathrm{Br}_{2}\right] \mathrm{BrCs}_{3}$. 


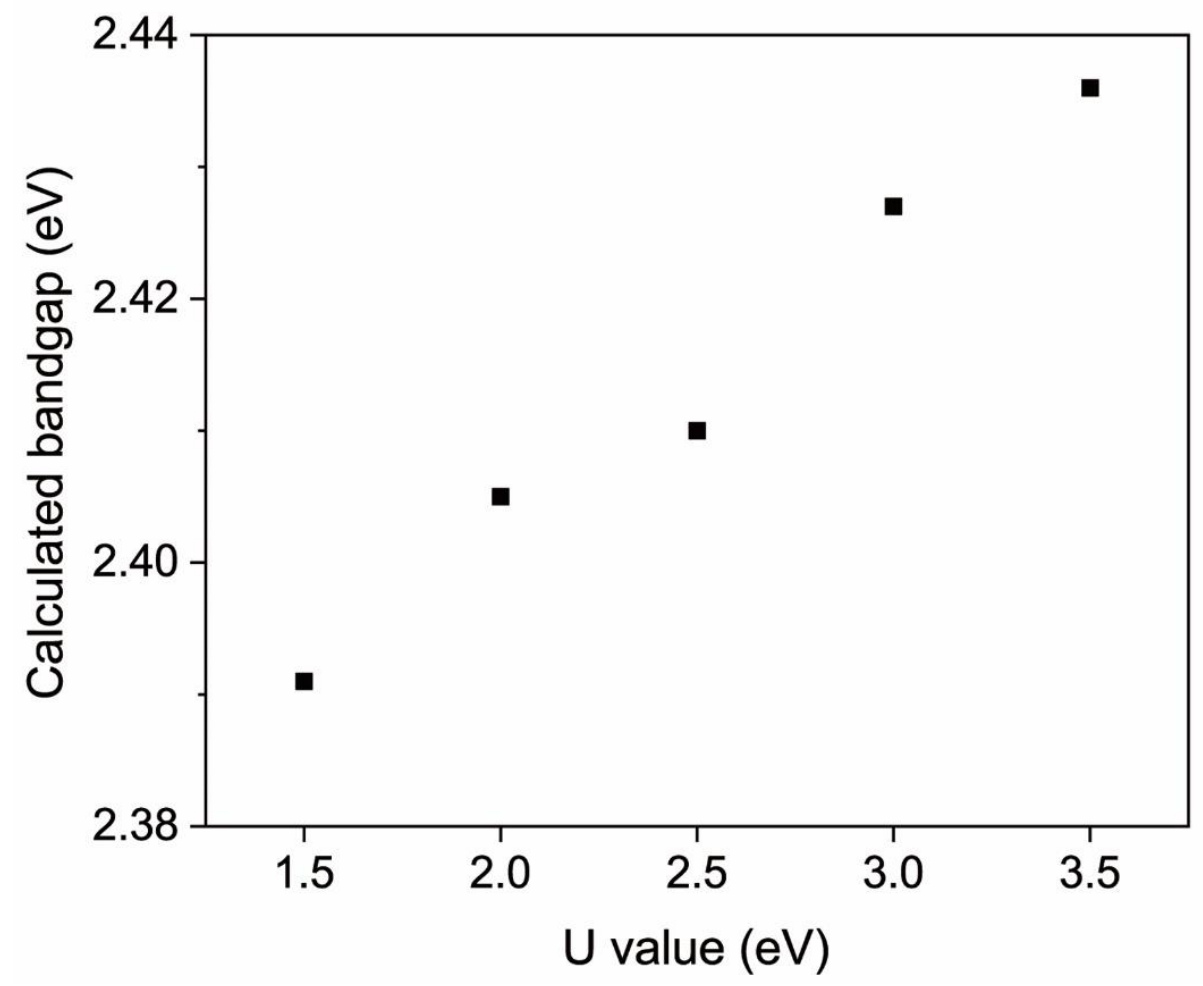

Figure S8. The dependence of the calculated band gap on the $U$ value. 

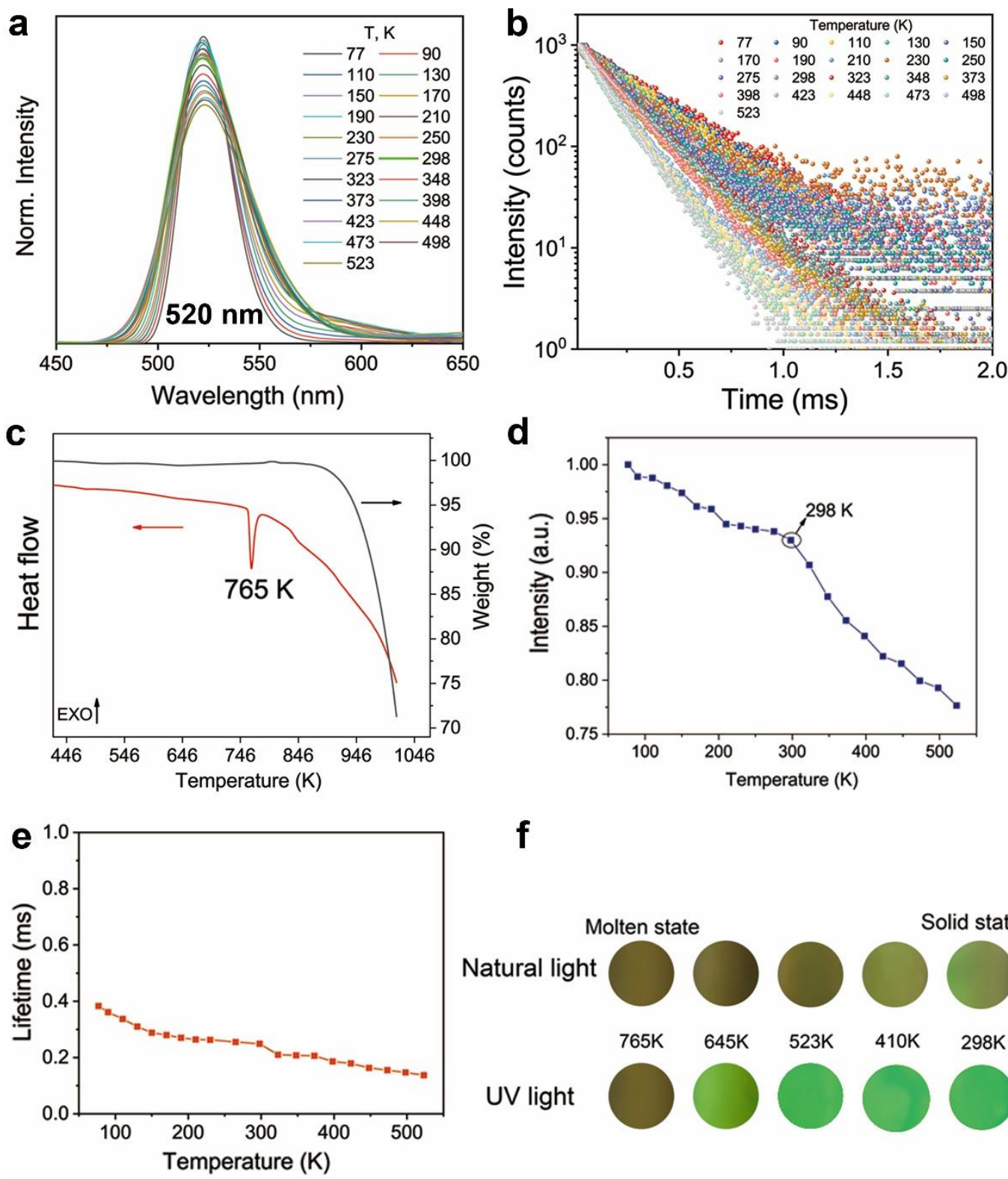

$\mathbf{f}$

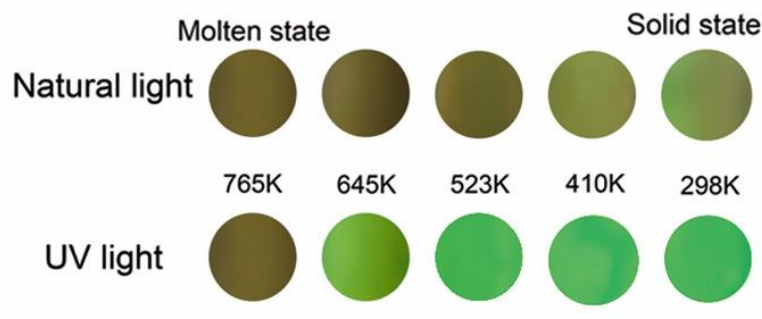

Figure S9. Effects of temperature on optical and thermal stability of single crystal

[ $\left.\mathbf{M n C l}_{2} \mathbf{B r}_{2}\right] \mathbf{B r C s} 3$. (a) Temperature-dependent PL spectra from $77 \mathrm{~K}$ to $523 \mathrm{~K}$. (b) Time-resolved PL decay curves from $77 \mathrm{~K}$ to $523 \mathrm{~K}$. (c) TGA (black line) of anti-perovskite $\left[\mathrm{MnCl}_{2} \mathrm{Br}_{2}\right] \mathrm{BrCs}_{3}$ $(5 \%$ loss, $943 \mathrm{~K})$ and DSC trace (red line) of anti-perovskite $\left[\mathrm{MnCl}_{2} \mathrm{Br}_{2}\right] \mathrm{BrCs}_{3}$ (scan rate: 10 ${ }^{\circ} \mathrm{C} / \mathrm{min}$ ). (d) PL intensity as a function of temperature from $77 \mathrm{~K}$ to $523 \mathrm{~K}$. (e) Lifetime as a function of temperature from $77 \mathrm{~K}$ to $523 \mathrm{~K}$. (f) Images of single crystal $\left[\mathrm{MnCl}_{2} \mathrm{Br}_{2}\right] \mathrm{BrCs}_{3}$ under natural light (up) and UV light (down) from the molten temperature to the room temperature. 

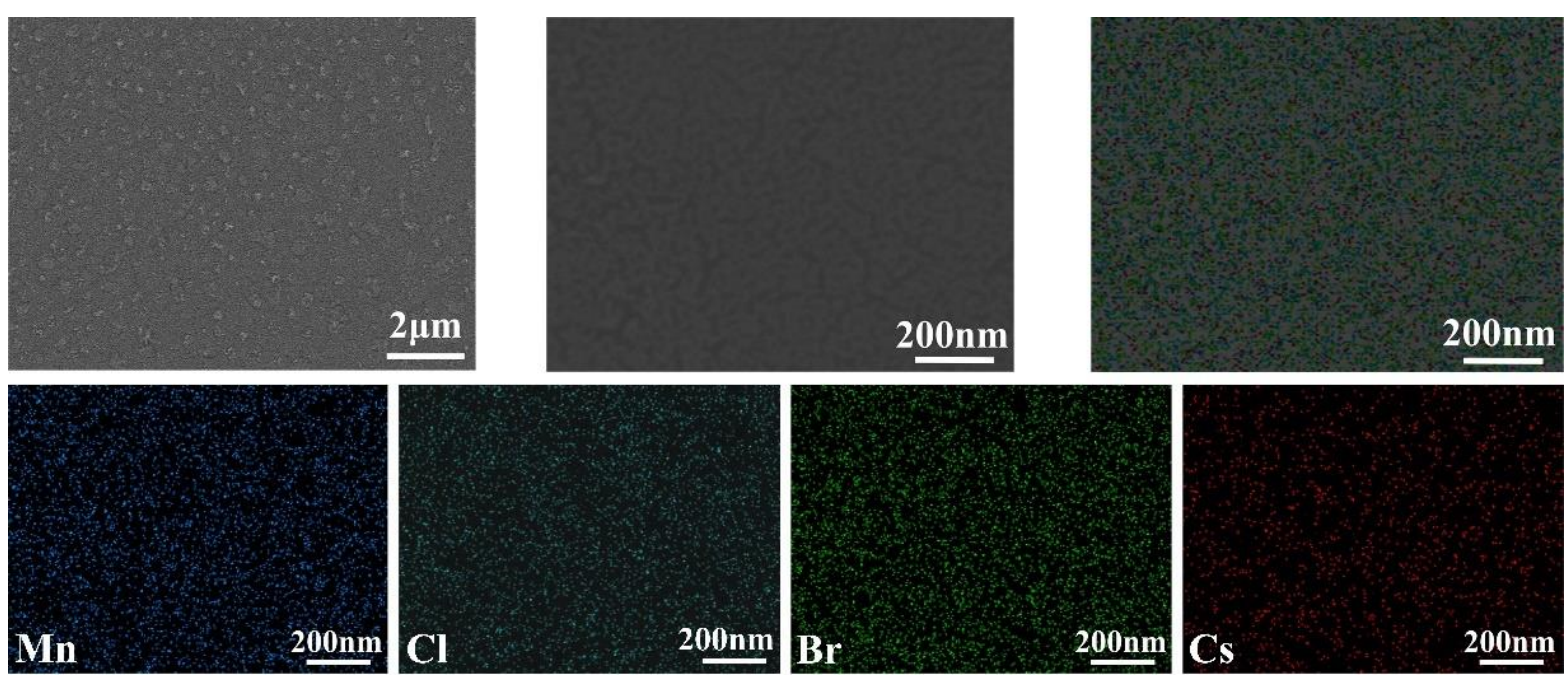

Figure S10. SEM images of $\left[\mathrm{MnCl}_{2} \mathrm{Br}_{2}\right] \mathrm{BrCs} 3$ film fabricated by the double-source thermal evaporation strategy. SEM images (greyscale) of $\left[\mathrm{MnCl}_{2} \mathrm{Br}_{2}\right] \mathrm{BrCs} 3$ film at different magnifications and elemental mapping images (in color). 


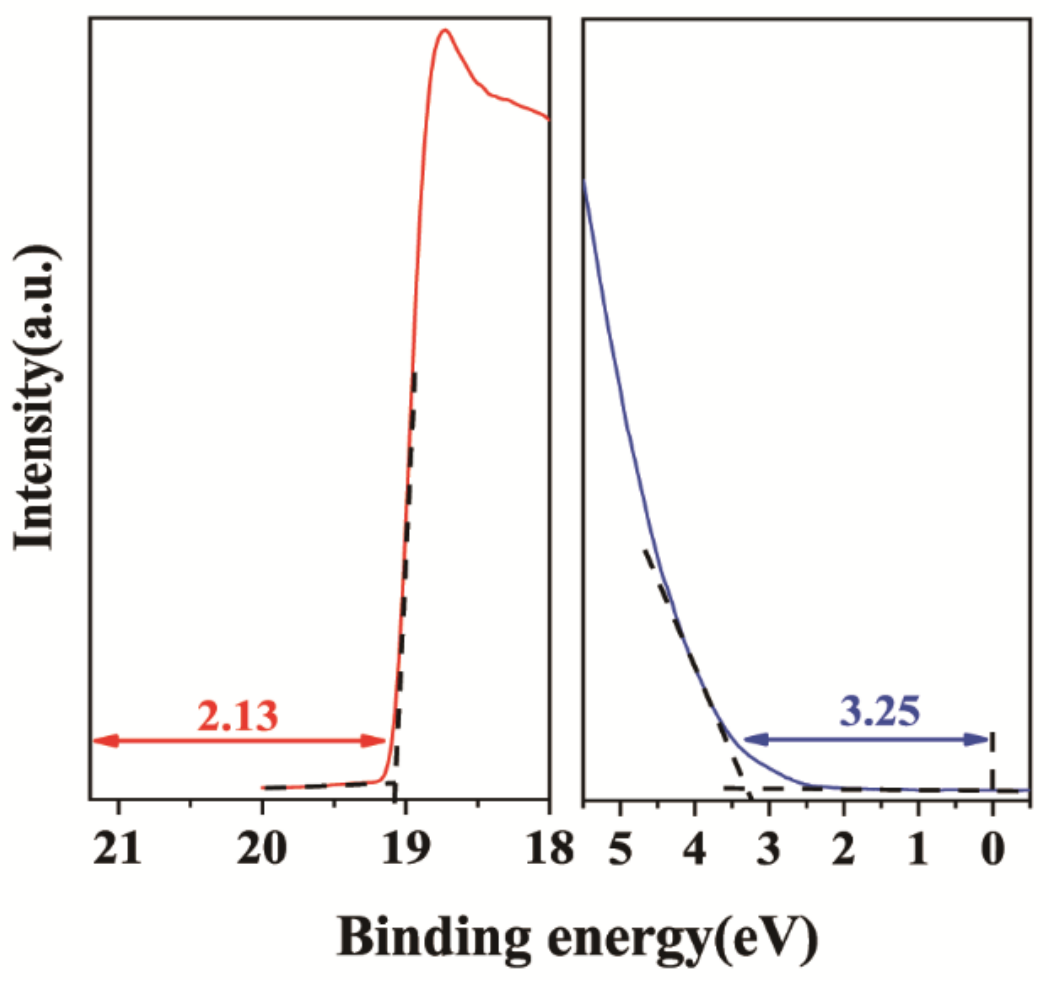

Figure S11. UPS data of film $\left[\mathrm{MnCl}_{2} \mathrm{Br}_{2}\right] \mathrm{BrCs3}$. The binding energy cutoff ( $\left.E_{\text {cutoff }}\right)$ is calculated by linear extrapolation to zero of the yield of secondary electrons. From the left figure, $E_{\text {cutoff }}$ $=19.07 \pm 0.03 \mathrm{eV}$. The onset of film relative to the Fermi energy of Au, $E_{\text {onset }}=3.25 \pm 0.0 .3 \mathrm{eV}$. The incident photon energy, $h v=21.2 \mathrm{eV}$. The valence band maximum energy (ЕvBM) is therefore determined by the formula, $\mathrm{E}_{\mathrm{VBM}}=\mathrm{h} v-\left(\mathrm{E}_{\text {cutoff- }} \mathrm{E}_{\text {onset }}\right)$. For film $\left[\mathrm{MnCl}_{2} \mathrm{Br}_{2}\right] \mathrm{BrCs}_{3}, E_{\mathrm{VBM}}=-5.38 \pm$ $0.06 \mathrm{eV}^{21}$ 


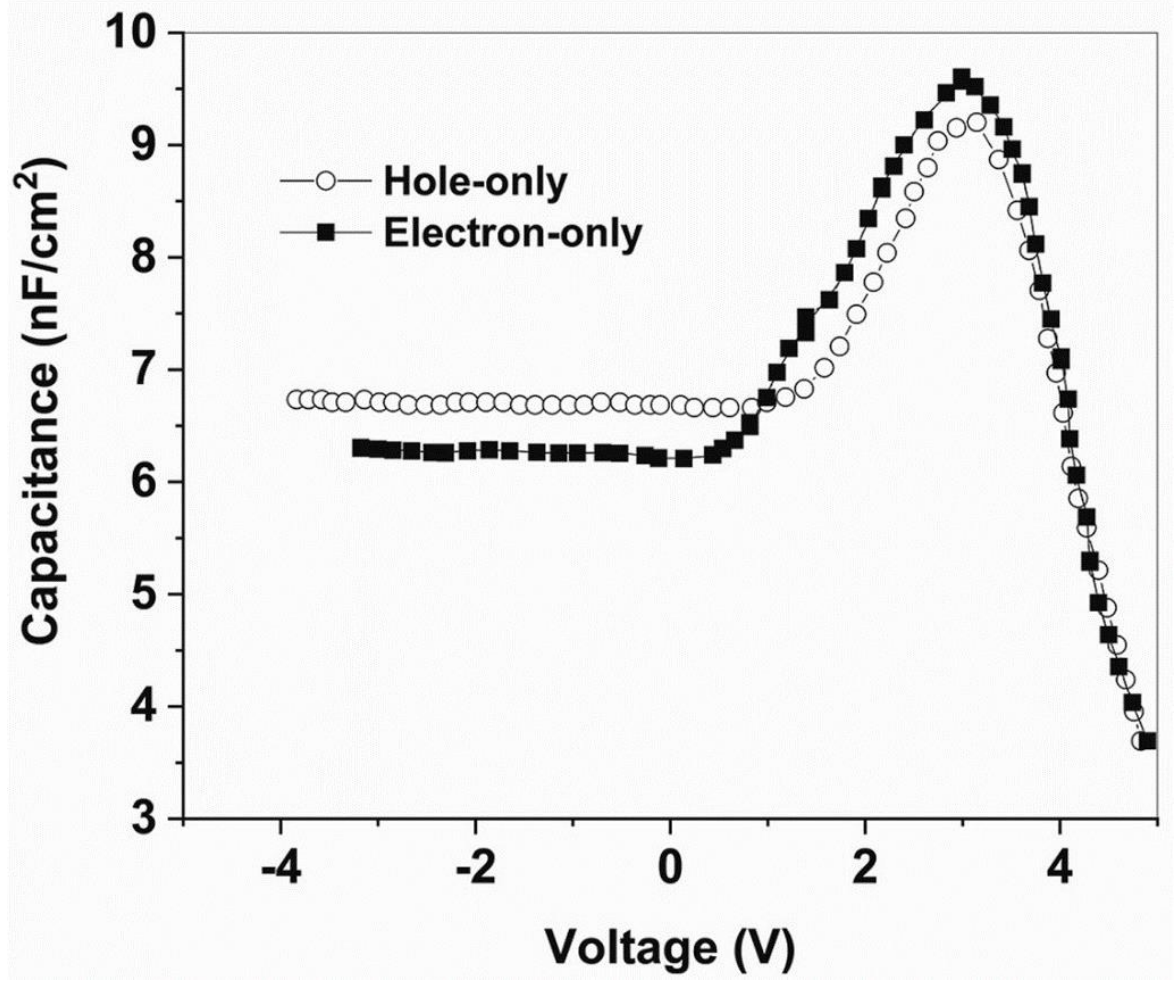

Figure S12. C-V characteristic of hole-only device and electron-only devices measured at $1 \mathrm{kHz}$ as a function of reversed and forward bias. The structure of hole-only device consists of ITO/PEDOT:PSS $(40 \mathrm{~nm}) /\left[\mathrm{MnCl}_{2} \mathrm{Br}_{2}\right] \mathrm{BrCs}_{3}(45 \mathrm{~nm}) / \mathrm{Au}(80 \mathrm{~nm})$, and the structure of electrononly device consists of ITO/TPBi $(40 \mathrm{~nm}) /\left[\mathrm{MnCl}_{2} \mathrm{Br}_{2}\right] \mathrm{BrCs}_{3}(45 \mathrm{~nm}) / \mathrm{TPBi}(40 \mathrm{~nm}) / \mathrm{Al}(100 \mathrm{~nm})$. The applied ac bias voltage is $50 \mathrm{mV}$. 


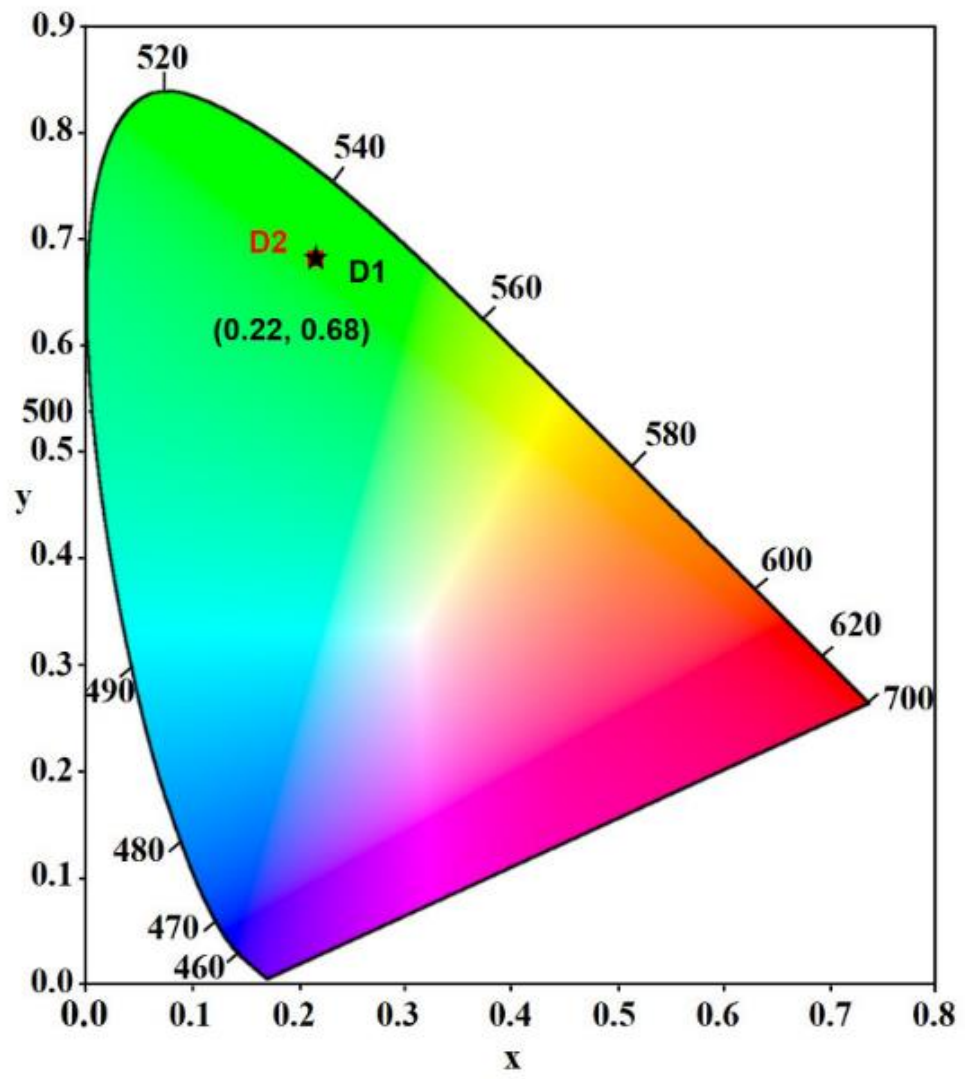

Figure S13. Chromaticity of the emission extracted from the EL spectra of D1 and D2. The CIE color coordinates are both located at $(0.22,0.68)$. 


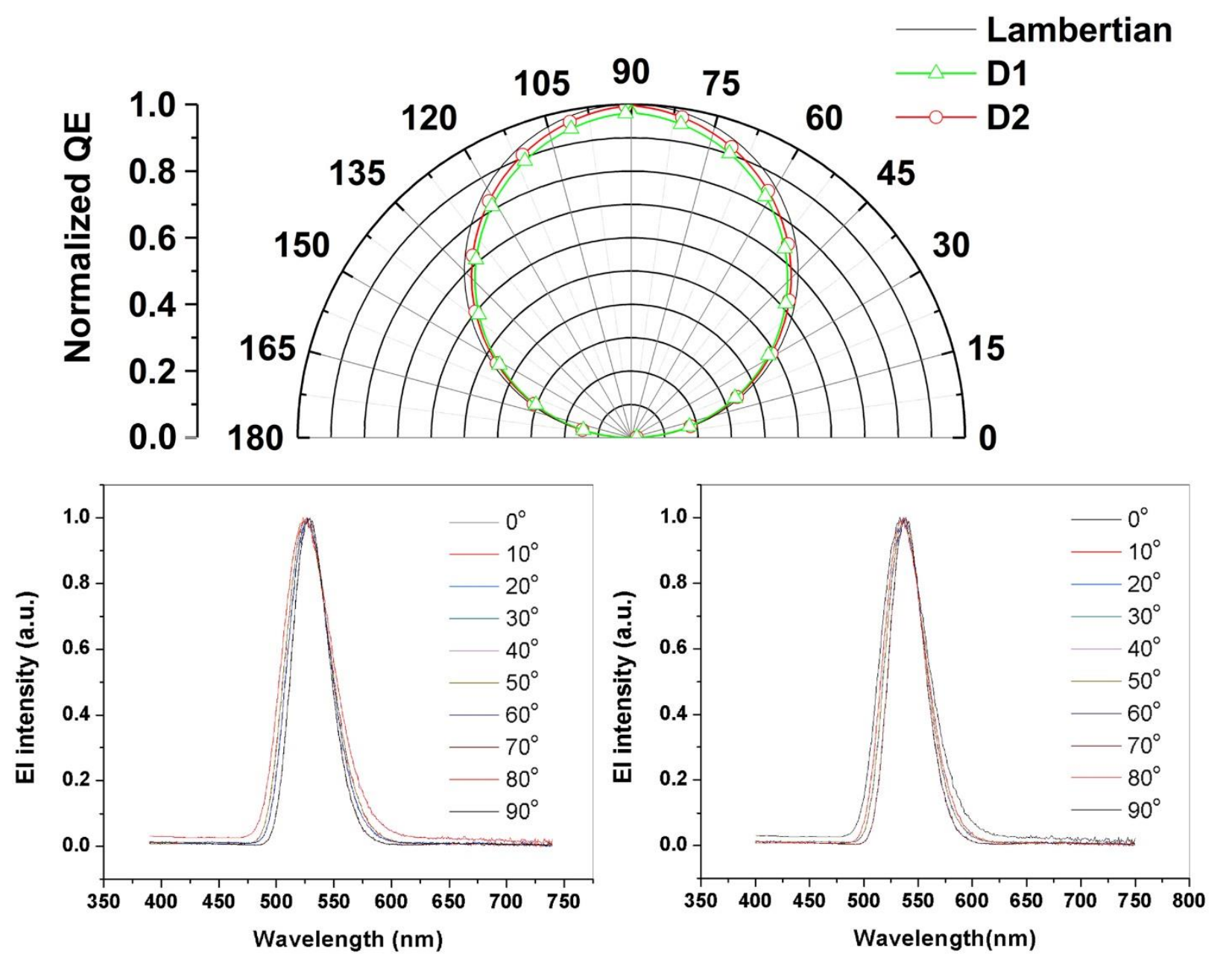

Figure S14. Optoelectronic characteristics of APeLEDs. The angular distribution of radiation intensity follows a Lambertian profile. The EL spectra of D1 (left) and D2 (right) at different viewing angles $\left(0,10,20,30,40,50,60,70,80\right.$, and $\left.90^{\circ}\right)$. 


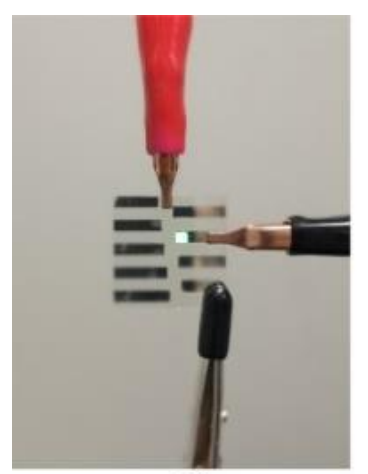

a

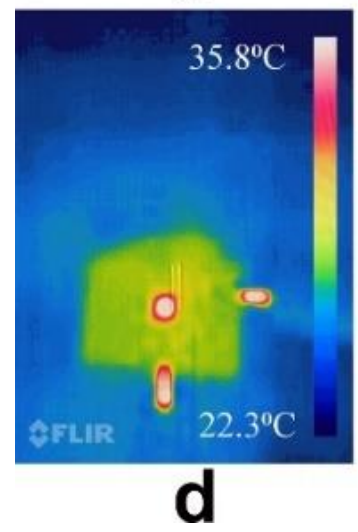

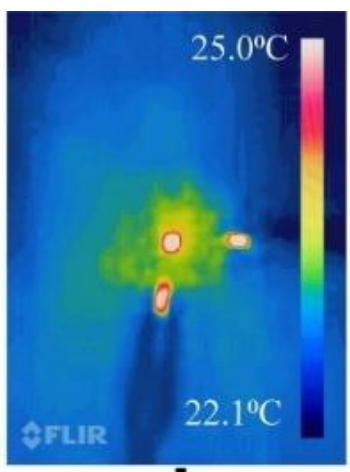

b

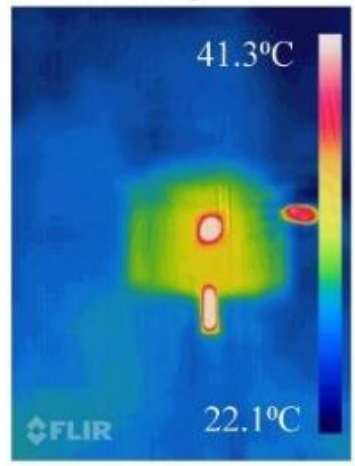

e

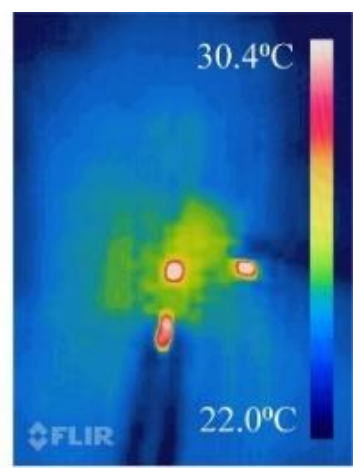

C

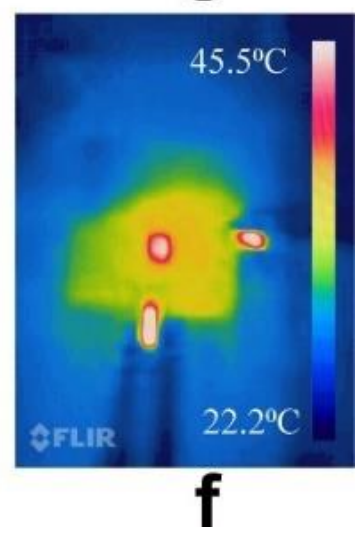

Figure S15. Temperature analysis of D1 during operation at the voltage of $5.0 \mathrm{~V}$ by the infrared thermal imager. (a) The image of the tested device under the natural light. (b)-(f) Spatial surface temperature glass substrate monitored at different time points $(30,60,100,200$, and 300 $\min )$. 
Table S10. Summary of the device performance of the lead-free perovskite and metal halide green LEDs.

\begin{tabular}{|c|c|c|c|c|c|}
\hline Emission materials & EL $\lambda_{\max }(\mathrm{nm})$ & $\operatorname{Lumi}_{\max }\left(\mathrm{cd} \mathrm{m}^{-2}\right)$ & $\begin{array}{l}\mathrm{EQE}_{\max } \\
(\%)\end{array}$ & $\begin{array}{l}\text { Lifetime } \\
(\mathrm{min})\end{array}$ & Refs. \\
\hline \multicolumn{6}{|c|}{ Lead-based doping and lead-free perovskite green LEDs } \\
\hline $\mathrm{CsCu}_{2} \mathrm{I}_{3}$ & 560 yellow & 10 & 0.02 & N/A & 22 \\
\hline $\mathrm{Cs}_{2} \mathrm{AgIn}_{0.6} \mathrm{Na}_{0.4} \mathrm{InCl}_{6} / \mathrm{UV}$ LED chips & (552) warm-white & 50 & $\mathrm{~N} / \mathrm{A}$ & $\begin{array}{l}10 \quad(50 \\
\left.\mathrm{mA} / \mathrm{cm}^{2}\right)\end{array}$ & 23 \\
\hline$(\mathrm{PEA})_{2} \mathrm{SnIBr}_{3}$ & 522 & $\mathrm{~N} / \mathrm{A}$ & $\mathrm{N} / \mathrm{A}$ & N/A & 24 \\
\hline$(\mathrm{PEA})_{2} \mathrm{SnI}_{2} \mathrm{Br}_{2}$ & 556 & $\mathrm{~N} / \mathrm{A}$ & N/A & N/A & 24 \\
\hline $\mathrm{PEA}_{0.2} \mathrm{Cs}_{0.4} \mathrm{MA}_{0.6} \mathrm{~Pb}_{0.7} \mathrm{Mn}_{0.3} \mathrm{Br}_{3}$ & $\sim 520$ & 97000 & 3.2 & 23 & 25 \\
\hline $\mathrm{CsPb}_{0.9} \mathrm{Sn}_{0.1} \mathrm{Br}_{3}$ & 519 & 1600 & 1.8 & N/A & 26 \\
\hline $\mathrm{CsPb}_{0.67} \mathrm{Sn}_{0.33} \mathrm{Br}_{3}$ & 517 & 12500 & 4.13 & N/A & 27 \\
\hline $\mathrm{CsPb}_{0.9} \mathrm{Sn}_{0.1} \mathrm{PbBr}_{3}$ & 518 & 5070 & N/A & N/A & 28 \\
\hline $\mathrm{CsPb}_{0.7} \mathrm{Sn}_{0.3} \mathrm{PbBr}_{3}$ & 508 & 5495 & N/A & N/A & 28 \\
\hline $\mathrm{CsPb}_{0.5} \mathrm{Sn}_{0.5} \mathrm{PbBr}_{3}$ & 504 & 2203 & N/A & N/A & 28 \\
\hline $\mathrm{CsPb}_{0.3} \mathrm{Sn}_{0.7} \mathrm{PbBr}_{3}$ & 501 & 1517 & N/A & N/A & 28 \\
\hline $\mathrm{CsPbBr}_{3}: \mathrm{Mn} 3.8 \mathrm{~mol} \%$ & $512-515$ & 9717 & 1.49 & $\mathrm{~N} / \mathrm{A}$ & 29 \\
\hline
\end{tabular}

\section{Lead-free metal halide green LEDs}

\begin{tabular}{llllll}
\hline $\mathrm{CsCuBr}_{2}$ & 527 & N/A & N/A & N/A & 30 \\
$\left(\mathrm{Ph}_{4} \mathrm{P}\right)_{2}\left[\mathrm{MnBr}_{4}\right]$ & $\sim 528$ & 2339 & 9.6 & N/A & 31 \\
$\mathrm{DBFDPO}-\mathrm{MnBr}_{2}$ & 552 & 393 & 10.49 & N/A & 32 \\
$\left(\mathrm{C}_{10} \mathrm{H}_{16} \mathrm{~N}\right)_{2} \mathrm{MnBr}_{4} / \mathrm{K}_{2} \mathrm{SiF}_{6}: \mathrm{Mn}^{4+} / \mathrm{InGaN}$ chips & $(518)$ white & N/A & N/A & N/A & 33 \\
$\mathrm{Cs}_{3} \mathrm{MnBr}_{5} / \mathrm{KSF}_{2} \mathrm{Mn}^{4+} / \mathrm{GaN}$ chips & $(520)$ white & N/A & N/A & N/A & 34 \\
$\beta-\mathrm{Cs}_{3} \mathrm{Cu}_{2} \mathrm{Cl}_{5} / \mathrm{UV}$ chips & $\sim 510$ & N/A & N/A & N/A & 35
\end{tabular}

\section{Lead-free anti-perovskite green LEDs}

\begin{tabular}{lccccc}
\hline$\left[\mathrm{MnCl}_{2} \mathrm{Br}_{2}\right] \mathrm{BrCs}_{3}$ & $\mathbf{5 2 0}$ & 3990 & 12.5 & 756 & This \\
work
\end{tabular}

N/A: The values were not reported in the references. 


\section{References}

(1) Pakhomov, V.; Fedorov, P.; Sadikov, G. Structure of $\mathrm{Cs}_{3} \mathrm{HgBr}_{5}$ Crystals. Kristallografiya 1978, $23(3), 615-616$.

(2) Clegg, W.; Brown, M. L.; Wilson, L. J. A. Tricaesium Tetrachloromercurate(II) Chloride. Acta Crystallogr. B 1976, 32 (10), 2905-2906.

(3) Sjövall, R.; Svensson, C. Crystal Structures of Four $\mathrm{Cs}_{3} \mathrm{MI}_{5}(\mathrm{M}=\mathrm{Mn}, \mathrm{Cd}, \mathrm{Hg})$ Phases and Structural Relationship among $\mathrm{Cs}_{3} \mathrm{MI}_{5}$ Compounds. Zeitschrift Fur Kristallographie - Z KRISTALLOGR 1997, 212 (10), 732-741.

(4) Kruglik, A.; Vasil'ev, A.; Grankina, V.; Kovalev, G. Crystal Structures of $\mathrm{Cs}_{3} \mathrm{CdBr}_{5}$ and $\left(\mathrm{NH}_{4}\right)_{2.34} \mathrm{Rb}_{0 .}{ }_{66} \mathrm{ZnBr}_{5}$. Soviet physics. Crystallography 1992, 37 (3), 303-305.

(5) Villars, P.; Cenzual, K.; Gladyshevskii, R.; Shcherban, O.; Dubenskyy, V.; Kuprysyuk, V.; Savysyuk, I.; Zaremba, R., Landolt-Börnstein. Springer-Verlag Berlin Heidelberg: 2012, 10.1007/978-3-642-22847-6_389.

(6) Müller, B. G. Neue Fluropalladate (II). Zeitschrift für anorganische und allgemeine Chemie 1982, $491(1), 245-252$.

(7) Goodyear, J.; Kennedy, D. Crystal Structure of $\mathrm{Cs}_{3} \mathrm{MnCl}_{5}$. Acta Crystallographica Section B: Structural Crystallography and Crystal Chemistry 1976, 32 (2), 631-632.

(8) Seifert, H. J.; Flohr, G. Lösungskalorimetrische Messungen an Doppelchloriden UndBromiden Des Zweiwertigen Mangans. Zeitschrift für anorganische und allgemeine Chemie 1977, $436(1), 244-252$.

(9) Amit, M.; Horowitz, A.; Ron, E.; Makovsky, J. Preparation and Crystal Structures of Some Compounds of the $\mathrm{A}_{3} \mathrm{BX}_{5}$ Type $\left(\mathrm{A}=\mathrm{Cs}, \mathrm{Tl}, \mathrm{NH}_{4}, \mathrm{~B}=\mathrm{Mn}, \mathrm{Fe}, \mathrm{Co}, \mathrm{X}=\mathrm{Cl}, \mathrm{Br}\right)$. Isr. J. Chem. 1973, 11 (6), 749-764. 
(10) Fejfarova, K.; Ouarsal, R.; El Bali, B.; Dušek, M.; Lachkar, M. Tricaesium Tetrachloridozincate (II) Chloride. Acta Crystallographica Section E: Structure Reports Online 2007, 63 (5), i136-i137.

(11) Heming, M.; Lehmann, G.; Henkel, G.; Krebs, B. Correlation of Zero Field Splittings and Site Distortions III. $\mathrm{MnBr}_{4}{ }^{2-}$ and Crystal Structure Refinements for $\mathrm{Rb}_{3} \mathrm{ZnBr}_{5}$ and $\mathrm{Cs}_{2} \mathrm{ZnBr}_{4}$. Zeitschrift für Naturforschung A 1981, 36 (3), 286-293.

(12) Kanishcheva, A.; Zaitseva, I. Y.; Kovalyova, I.; Mikhailov, Y. N. Synthesis and Crystal Structure of $\mathrm{Cs}_{3} \mathrm{ZnBr}_{5}$. Russian Journal of Inorganic Chemistry 2010, 55 (12), 1882-1887.

(13) Friese, K.; Madariaga, G.; Breczewski, T. Tricaesium Tetraiodozincate (II) Iodide, $\mathrm{Cs}_{3} \mathrm{ZnI}_{5}$. Acta Crystallographica Section C: Crystal Structure Communications 1998, 54 (12), 1737-1739. (14) Sassmannshausen, M.; Lutz, H. D. Caesium Nickel (II) Trichloride, $\mathrm{CsNiCl}_{3}$, and Tricaesium Nickel (II) Pentachloride, $\mathrm{Cs}_{3} \mathrm{NiCl}_{5}$. Acta Crystallographica Section C: Crystal Structure Communications 1998, 54 (6), 704-706.

(15) Des Phasendiagramms, D.-U.; Des Co, D. Zur Existenz Einer Verbindung $\mathrm{Cs}_{3} \mathrm{CoI}_{5}$ Und Ihrer Kristallstruktur (1). Journal of Solid State Chemistry 1978, 26 (4), 397-399.

(16) Sosman, L. P.; López, A.; Camara, A. R.; Pedro, S. S.; Carvalho, I. C. S.; Cella, N. Optical and Structural Properties of $\mathrm{Zn}_{2} \mathrm{TiO}_{4}: \mathrm{Mn}^{2+} . J$. Electron. Mater. 2017, 46 (12), 6848-6855.

(17) Perdew, J. P.; Burke, K.; Ernzerhof, M. Generalized Gradient Approximation Made Simple. Phys. Rev. Lett. 1996, 77 (18), 3865-3868.

(18) Dudarev, S. L.; Botton, G. A.; Savrasov, S. Y.; Humphreys, C. J.; Sutton, A. P. ElectronEnergy-Loss Spectra and the Structural Stability of Nickel Oxide: An Lsda+U Study. Phys. Rev. $B$ 1998, 57 (3), 1505-1509. 
(19) Segall, M. D.; Lindan, P. J. D.; Probert, M. J.; Pickard, C. J.; Hasnip, P. J.; Clark, S. J.; Payne, M. C. First-Principles Simulation: Ideas, Illustrations and the Castep Code. J. Phys.: Condens. Matter 2002, 14 (11), 2717-2744.

(20) Vanderbilt, D. Soft Self-Consistent Pseudopotentials in a Generalized Eigenvalue Formalism. Phys. Rev. B 1990, 41 (11), 7892-7895.

(21) Zheng, L.; Zhu, T.; Xu, W.; Liu, L.; Zheng, J.; Gong, X.; Wudl, F. Solution-Processed Broadband Polymer Photodetectors with a Spectral Response of up to $2.5 \mu \mathrm{m}$ by a Low Bandgap Donor-Acceptor Conjugated Copolymer. J. Mater. Chem. C 2018, 6 (14), 3634-3641.

(22) Liu, N.; Zhao, X.; Xia, M.; Niu, G.; Guo, Q.; Gao, L.; Tang, J. Light-Emitting Diodes Based on All-Inorganic Copper Halide Perovskite with Self-Trapped Excitons. J. Semicond. 2020, 41 (5), 052204.

(23) Luo, J.; Wang, X.; Li, S.; Liu, J.; Guo, Y.; Niu, G.; Yao, L.; Fu, Y.; Gao, L.; Dong, Q.; Zhao, C.; Leng, M.; Ma, F.; Liang, W.; Wang, L.; Jin, S.; Han, J.; Zhang, L.; Etheridge, J.; Wang, J.; Yan, Y.; Sargent, E. H.; Tang, J. Efficient and Stable Emission of Warm-White Light from LeadFree Halide Double Perovskites. Nature 2018, 563 (7732), 541-545.

(24) Lanzetta, L.; Marin-Beloqui, J. M.; Sanchez-Molina, I.; Ding, D.; Haque, S. A. TwoDimensional Organic Tin Halide Perovskites with Tunable Visible Emission and Their Use in Light-Emitting Devices. ACS Energy Lett. 2017, 2 (7), 1662-1668.

(25) Gangishetty, M. K.; Sanders, S. N.; Congreve, D. N. $\mathrm{Mn}^{2+}$ Doping Enhances the Brightness, Efficiency, and Stability of Bulk Perovskite Light-Emitting Diodes. ACS Photonics 2019, 6 (5), $1111-1117$. 
(26) Deng, J.; Wang, H.; Xun, J.; Wang, J.; Yang, X.; Shen, W.; Li, M.; He, R. Room-Temperature Synthesis of Excellent-Performance $\mathrm{CsPb}_{1-\mathrm{x}} \mathrm{Sn}_{\mathrm{x}} \mathrm{Br}_{3}$ Perovskite Quantum Dots and Application in Light Emitting Diodes. Mater. Design 2020, 185 (5), 108246.

(27) Wang, H.-C.; Wang, W.; Tang, A.-C.; Tsai, H.-Y.; Bao, Z.; Ihara, T.; Yarita, N.; Tahara, H.; Kanemitsu, Y.; Chen, S.; Liu, R.-S. High-Performance $\mathrm{CsPb}_{1-\mathrm{x}} \mathrm{Sn}_{\times} \mathrm{Br}_{3}$ Perovskite Quantum Dots for Light-Emitting Diodes. Angew. Chem. Int. Ed. 2017, 56 (44), 13650-13654.

(28) Zhang, X.; Cao, W.; Wang, W.; Xu, B.; Liu, S.; Dai, H.; Chen, S.; Wang, K.; Sun, X. W. Efficient Light-Emitting Diodes Based on Green Perovskite Nanocrystals with Mixed-Metal Cations. Nano Energy 2016, 30, 511-516.

(29) Zou, S.; Liu, Y.; Li, J.; Liu, C.; Feng, R.; Jiang, F.; Li, Y.; Song, J.; Zeng, H.; Hong, M.; Chen, X. Stabilizing Cesium Lead Halide Perovskite Lattice through Mn(II) Substitution for Air-Stable Light-Emitting Diodes. J. Am. Chem. Soc. 2017, 139 (33), 11443-11450.

(30) Li, T.; Mo, X.; Peng, C.; Lu, Q.; Qi, C.; Tao, X.; Ouyang, Y.; Zhou, Y. Distinct Green Electroluminescence from Lead-Free $\mathrm{CsCuBr}_{2}$ Halide Micro-Crosses. Chem. Commun. 2019, 55 (31), 4554-4557.

(31) Xu, L.-J.; Sun, C.-Z.; Xiao, H.; Wu, Y.; Chen, Z.-N. Green-Light-Emitting Diodes Based on Tetrabromide Manganese(II) Complex through Solution Process. Adv. Mater. 2017, 29 (10), 1605739.

(32) Qin, Y.; Tao, P.; Gao, L.; She, P.; Liu, S.; Li, X.; Li, F.; Wang, H.; Zhao, Q.; Miao, Y.; Huang, W. Designing Highly Efficient Phosphorescent Neutral Tetrahedral Manganese(II) Complexes for Organic Light-Emitting Diodes. Adv. Opt. Mater. 2019, 7 (2), 1801160. 
(33) Zhou, G.; Liu, Z.; Huang, J.; Molokeev, M. S.; Xiao, Z.; Ma, C.; Xia, Z. Unraveling the nearUnity Narrow-Band Green Emission in Zero-Dimensional $\mathrm{Mn}^{2+}$-Based Metal Halides: A Case Study of $\left(\mathrm{C}_{10} \mathrm{H}_{16} \mathrm{~N}\right)_{2} \mathrm{Zn}_{1-\mathrm{x}} \mathrm{Mn}_{\mathrm{x}} \mathrm{Br}_{4}$ Solid Solutions. J. Phys. Chem. Lett. 2020, 11 (15), 5956-5962. (34) Su, B.; Molokeev, M. S.; Xia, Z. $\mathrm{Mn}^{2+}$-Based Narrow-Band Green-Emitting $\mathrm{Cs}_{3} \mathrm{MnBr}_{5}$ Phosphor and the Performance Optimization by $\mathrm{Zn}^{2+}$ Alloying. J. Mater. Chem. C 2019, 7 (36), $11220-11226$.

(35) Zhang, R.; Mao, X.; Zheng, D.; Yang, Y.; Yang, S.; Han, K. A Lead-Free All-Inorganic Metal Halide with Near-Unity Green Luminescence. Laser Photonics Rev. 2020, 14 (5), 2000027. 